Pacific Journal of Mathematics

A CHARACTERIZATION OF GROUPS IN TERMS OF TH 


\title{
A CHARACTERIZATION OF GROUPS IN TERMS OF THE DEGREES OF THEIR CHARACTERS II
}

\author{
I. M. IsaAcs AND D. S. Passman
}

In this paper we continue our study of the relationship between the structure of a finite group $G$ and the set of degrees of its irreducible complex characters. The following hypotheses on the degrees are considered: (A) $G$ has r.x. $e$ for some prime $p$, i.e. all the degrees divide $p^{e},(\mathrm{~B})$ the degrees are linearly ordered by divisibility and all except 1 are divisible by exactly the same set of primes, (C) $G$ has a.c. $m$, i.e., all the degrees except 1 are equal to some fixed $m,(D)$ all the degrees except 1 are prime (not necessarily the same prime) and $(E)$ all the degrees except 1 are divisible by $p^{e}>p$ but none is divisible by $p^{e+1}$. In each of these situations, group theoretic information is deduced from the character theoretic hypothesis and in several cases complete characterizations are obtained.

In situation (A), the greater complexity which can occur when $e \geqq p$ is explored and a conjecture concerning $p$-groups with $e<p$ is studied and certain cases of it are proved. Detailed statements are made about groups $G$ satisfying (B) for which the common set of prime divisors of the degrees does not consist of a single prime for which $G$ has a nonabelian $\mathfrak{S}_{p}$ subgroup. These results are applied to situation (C), groups with a.c. $m$, and such groups are completely characterized when $m$ is not a prime power corresponding to a nonabelian Sylow subgroup. If $m=p^{e}$ and an $\mathfrak{S}_{p}$ of $G$ is nonabelian then it is shown that $G$ must be nilpotent unless $e=1$ (in which case $G$ has r.x. 1 and has been completely characterized in [2]). This reduces the study of groups with a.c. $m$ to $p$-groups and it is shown that a $p$-group $G$ with a.c. $p^{e}$ must have an abelian normal subgroup of index $p^{e}$ unless $G$ has class 2 or 3 . Further information is obtained about these "special" class 2 and 3 groups. It is also shown that if $e>1$ then $G$ must have class $\leqq p$.

Groups satisfying hypothesis (D) are completely characterized and it is shown that in this case there are at most two degrees different from 1. Finally it is shown that if $G$ satisfies hypothesis (E) and has a nonabelian $\Im_{p}$ subgroup then $G$ is nilpotent and has a.c. $p^{e}$. In all the situations considered in this paper, the group in question is shown to be solvable.

We use here the notation and terminology of [2].

1. Groups with r.x. $(p-1)$. In [4] we classified all groups with 
r.x.2. As it turned out, in that study the prime $p=2$ played a special role. It now appears that in the general classification of groups with r.x. $e$ those primes $p$ with $p \leqq e$ will again play a special role. In the other direction, this means that groups $G$ with r.x. $e$ and $p>e$ are somehow better behaved than the others. In this section we will attempt to justify this last statement.

Let $G$ have r. x. $e$ but not r.x. $(e-1)$. Then we say that $e=e(G)$ is the character exponent of $G$. If $G$ has a normal subgroup $N$ of index $p$ with $e(N)=e(G)-1$, then in terms of the characterization problem, $G$ is trivial. We say that such groups are imprimitive. Otherwise $G$ is primitive. We note that since all groups with r.x. $e$ are $M$-groups this terminology causes no confusion.

The following result handles the nonnilpotent case. It shows moreover that the nonnilpotent exceptional group of [4](Theorem A (ii)) belongs in some sense to a series of such groups.

THEOREM 1.1. Let $e(G)=e$ and let $\Im_{p^{\prime}}(G)$ be noncentral. If $p \neq 2$ and $p$ is not a Mersenne prime or if $p>e$, then $G$ is imprimitive. If $p=e$, then $G$ is imprimitive unless $G / 3(G) \cong G_{0}$ where $G_{0}=\left(V \times{ }_{0} C\right) \sim C,|C|=p, V$ is elementary abelian and either $p=$ $2,|V|=3$ or $p=2^{\alpha}-1$ is a Mersenne prime and $|V|=2^{\alpha}$.

Conversely if $e(G)=e$ and $G / \mathcal{B}(G) \cong G_{0}$ given above, then $p=e$ and $G$ is primitive.

The lemma below is well known.

LEMMA 1.2. Let $\pi$ be a set of primes and let arbitrary group $G$ have a normal abelian $\mathfrak{S}_{\pi}$ subgroup $A$. Then $A=Z \times B$ where $Z$ and $B$ are characteristic in $G$ and $Z=3(G) \cap A$.

Proof. Clearly $A$ is characteristic in $G$ and $G / A$ acts on $A$. Let $\theta$ be the endomorphism of $A$ which is given by $\theta(a)=\prod_{x \in G / A} a^{x}$. Clearly $K=\operatorname{ker} \theta$ and $I=$ image $\theta$ are characteristic subgroups of $G$ and $|K| \cdot|I|=|A|$. If $Z=3(G) \cap A$, then we see easily than $Z \supseteqq I$ and $Z \cap K=\langle 1\rangle$. The latter uses the fact that $A$ is an $\mathfrak{S}_{\pi}$ subgroup of $G$. Hence $Z=I$ and $A=Z \times K$.

Proof of Theorem 1.1. Let $H=\mathfrak{S}_{p^{\prime}}(G)$ and $P=G / \mathfrak{S}(H)$. Let $\hat{H}$ be the group of linear characters of $H$ and let $G_{1}=\hat{H} \times{ }_{o} P$ where $P$ acts faithfully in the natural manner on $\hat{H}$. If there exists $\lambda \in \hat{H}$ with $\mathfrak{E}_{P}(\lambda)=\langle 1\rangle$ then choose $N$ with $N \supseteqq \mathfrak{S}(H)$ and $[G: N]=p$. By [5] $(\S 3$, in particular the proofs of Theorems 3.1 and 3.2$), e(N)=$ $e(G)-1$. Now this occurs by Corollary 2.4 (i) of [5] if $p \neq 2$ and $p$ is not a Mersenne prime or if $p>e(G)$. It also occurs for $p=e(G)$ 
unless $G_{1}$ has $G_{0}$ as a homomorphic image. This follows by Theorem 2.1 of [5] and a slight modification of Lemma 1.2 of [5] since we do not have to look at subgroups here. We consider this last possibility. Assume $G$ is primitive.

Now $P$ has as a homomorphic imape $P_{0}$, a Sylow $p$-subgroup of $G_{0}$. Since $\left[P_{0}: P_{0}^{\prime}\right]=p^{2}$ we see that $P_{0}$ has a nonabelian group of order $p^{3}$ as a homomorphic image. Thus there exists $K \triangle G$ with $K \supseteqq \mathfrak{S}(H)$ and $G / K$ nonabelian of order $p^{3}$. By [2](p. 885, equation * with $m=1$ ) $G$ has a normal subgroup $N$ with $K<N<G,[G: N]=p^{2}$ and $e(N) \leqq e(G)-1$. Since $p=e>e(N)$ we conclude by [5] that

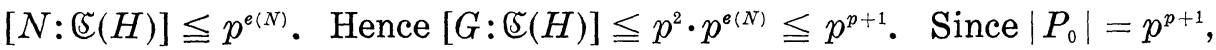
it follows that $[G: \mathbb{E}(H)]=p^{p+1}$ and $P \cong P_{0}$.

Let $W$ be the kernel of the homomorphism $G_{1} \rightarrow G_{0}$. By the above $W \subseteq \hat{H}$. We show that $W$ is central in $G_{1}$. Let $w \in W$ and suppose that $\mathfrak{S}_{G_{1}}(w)<G_{1}$. It is easy to see in $G_{0}$ that there exists $\bar{a}, \bar{b} \in \hat{H} / W$ such that $\left|\mathfrak{E}_{P_{0}}(\bar{a})\right|=\left|\mathfrak{S}_{P_{0}}(\bar{b})\right|=p$ and $P_{0}=\left\langle\mathfrak{S}_{P_{0}}(\bar{a}), \mathfrak{夭}_{P_{0}}(\bar{b})\right\rangle$. Thus since $G_{1}$ acts on $\hat{H} / W$ and $\mathfrak{夭}_{G_{1}}(w)<G_{1}$ we cannot have both $\mathfrak{夭}_{G_{1}}(w) \supseteqq \widetilde{夭}_{G_{1}}(\bar{a})$ and $\mathfrak{夭}_{G_{1}}(\bar{b})$. Say $\mathfrak{夭}_{G_{1}}(w) \nsupseteq \mathfrak{S}_{G_{1}}(\bar{a})$. Since $\left|\mathfrak{S}_{P_{0}}(\bar{a})\right|=p$ we have $\mathfrak{夭}_{G_{1}}(w) \cap$ $\mathfrak{夭}_{G_{1}}(\bar{a})=\hat{H}$. Now $p$-group $\mathfrak{夭}_{G_{1}}(\bar{a}) / \hat{H}$ permutes the elements of the coset $a W=\bar{a}$ and $|a W|$ is prime to $p$. Hence we can choose an element $a \in a W$ which is centralized by $\mathfrak{\Im}_{G_{1}}(\bar{a})$. Consider $v=a w \in \hat{H}$. If $x \in \mathfrak{S}_{G_{1}}(v)$ then $x \in \mathfrak{S}_{G_{1}}(\bar{v})=\mathfrak{E}_{G_{1}}(\bar{a})$. Thus $x$ centralizes $\bar{a}$ and hence $x \in \mathfrak{G}_{G_{1}}(\bar{a}) \cap \mathfrak{S}_{G_{1}}(w)=\hat{H}$. Therefore $\mathfrak{S}_{G_{1}}(v)=\hat{H}$ and this is a contradiction since $G$ is primitive. Thus $W$ is central in $G_{1}$ and since $G_{1} / W \cong$ $G_{0}, W=3\left(G_{1}\right)$. By Lemma $1.2, \hat{H}=W \times R$ where $R \triangle G_{1}$ and $R P \cong G_{0}$.

Since $\hat{H}=W \times R$ we have $H=Q \times Z$. All linear characters of $Z$ are fixed by $P$ and hence $Z$ is central in $G$. Also $\hat{Q} P \cong G_{0}$ and from the nature of $G_{0}$ we see easily that $Q P \cong G_{0}$ Moreover $\widetilde{夭}_{G}(H)=$ $Q \times Z \times S$ where $S=\mathfrak{S}_{p}(\mathfrak{S}(H))$. We show now that $S$ is central in $G$.

Choose $\lambda, \mu \in \hat{Q}$ with $\left|T_{P}(\lambda)\right|=\left|T_{P}(\mu)\right|=p$ and $P=\left\langle T_{P}(\lambda), T_{P}(\mu)\right\rangle$. Let $\varphi$ be an irreducible character of $S$. View $\lambda, \mu$ and $\phi$ as characters of $\mathfrak{C}(H)$. Let $\chi$ be a constituent of $(\lambda \varphi)^{*}$ so that $\chi \mid \mathfrak{\subseteq}(H)=\alpha \sum_{1}^{t}(\lambda \varphi)_{i}$. Clearly $T(\lambda \varphi)=T(\lambda) \cap T(\varphi)$ so $t \geqq p^{p}$ and $p^{p} \geqq \operatorname{deg} \chi=a t \operatorname{deg} \varphi \geqq p^{p}$ $\operatorname{deg} \varphi$. Thus $\operatorname{deg} \varphi=1$ and $t=p^{p}$. This shows that $S$ is abelian and that $T(\varphi) \supseteqq T(\lambda)$. Similary $T(\varphi) \supseteqq T(\mu)$ and hence $T(\varphi)=G$. Therefore $S$ is central in $G$ and $3(G)=Z \times S$. Hence $G / 3(G) \cong Q P \cong G_{0}$ and the result follows.

We show conversely that all the exceptional groups discussed have $e(G)=p$ and are primitive. Let $A=\mathfrak{C}(H)$. Since $G / 3(G) \cong G_{0}$ we see that $A=H 3(G)$ is abelian since $H$ is abelian. Also $[G: A]=p^{p+1}$. Let $\chi$ be an irreducible character of $G$ and $\chi \mid A=a \sum_{1}^{t} \lambda_{i}$. Then $\operatorname{deg} \chi=a t$ and $a^{2} t \leqq[G: A]=p^{p+1}$ by Lemma 1.2 of [2]. Thus deg $\chi \leqq$ $p^{p+1}$ and if $\operatorname{deg} \chi=p^{p+1}$ then $a=1$ and $t=p^{p+1}$. The latter implies that for $\lambda=\lambda_{1}$ we have $T(\lambda)=A$. We ishow that this is not the 
case. Let $\lambda \mid B(G)=\mu$. Then $\lambda$ is a constituent of $\tilde{\mu}$ (induction to $A$ ) and $G / A$ permutes the linear constituents of $\tilde{\mu}$ since $3(G)$ is central. Now $G / A$ is a $p$-group and $\operatorname{deg} \tilde{\mu}$ is prime to $p$ so there exists a constituent $\eta$ of $\tilde{\mu}$ which is fixed by $G / A$. Since $\lambda / 3(G)=\eta / 3(G)$ it follows that $\lambda=\eta \xi$ where $\xi$ is a character with $\xi \mid 3(G)=1$. By properties of $G_{0}, T(\xi)>A$ and since $T(\eta)=G$, it follows that $T(\eta \xi)>A$. Thus $e(G) \leqq p$. Since $e\left(G_{0}\right)=p$ we have $e(G)=p$.

Suppose $G$ is imprimitive. Let $N \triangle G$ with $[G: N]=p$ and $e(N)=p-1$. Let $\chi$ be a character of $G$ of degree $p^{p}$. Since $e(N)=$ $p-1$ we have $\chi=\phi^{*}$ for some irreducible character $\varphi$ of $N$. This shows that $N \supseteqq 3(G)$. Clearly $N \supseteqq \Im_{p^{\prime}}(G)=H$ and therefore $N \supseteqq$ $H 3(G)=A$. Since $p>e(N)$ and $\mathfrak{夭}_{N}(H)$ is abelian, it follows from $\S 3$ of [5] that $[N: A]=p^{e(N)}$. Hence $[G: A]=p^{e(N)+1}=p^{p}$, a contradiction. This completes the proof.

We now study $p$-groups with r.x. $e$ and $p>e$. Here our results are not conclusive.

Let $p$-group $G$ have $e(G)=e$. We set $\Omega(G)$, the character kernel of $G$ equal to $\Omega(G)=\cap \operatorname{ker} \theta$ where $\theta$ runs over all irreducible characters of $G$ of $G$ of degree $p^{e}$. If $\Omega(G)=\langle 1\rangle$, we say $G$ is character regular. In [6](Corollary 2 with $n=p^{e-1}$ ) we showed that $|\Omega(G)| \leqq \frac{1}{2}\left(2 p^{e-1}\right)$ ! We conjecture that if $p>e(G)$ than $G$ is character regular. Reasons for studying this property can be seen in the following result.

Proposition 1.3. Let $G$ be a $p$-group with $e(G)=e$.

(i) Let $N \triangle G$ with $e(N)=e$. If $N$ is character regular then $3(N) \subseteq 3(G)$.

(ii) Suppose $G$ is primitive and every maximal subgroup is character regular. If $\zeta \in G-3(G)$, then $\left[G:(\zeta(\zeta)] \geqq p^{2}\right.$. Thus if $J$ is a central subgroup of $G$ of order $p$, then $3(G / J)=3(G) / J$.

Proof. In (i) suppose $3(N) \nsubseteq 3(G)$. Then we can choose $x \in(G, 3(N))$ with $x \neq 1$. Since $N \triangle G, x \in N$. Now $N$ is character regular so there exists irreducible character $\varphi$ of $N$ with $\operatorname{deg} \varphi=p^{e}$ and $x \notin \operatorname{ker} \varphi$. Let $\chi$ be and irreducible constituent of $\varphi^{*}$. Since $\operatorname{deg} \chi \leqq p^{e}$ we have clearly $\chi \mid N=\varphi$. Thus $3(N)$ is central in the representation associated with $\chi$ and $(G, 3(N)) \leqq \operatorname{ker} \chi \cap N=\operatorname{ker} \varphi$, a contradiction.

We consider (ii). Since $\zeta \notin 3(G)$ we have $[G:(\zeta(\zeta)] \geqq p$. If $[G: \mathfrak{G}(\zeta)]=p$, let $N=\mathfrak{S}(\zeta)$. Then $N \triangle G, N$ is character regular and $e(N)=e(G)$ since $G$ is primitive. By (i), $3(N) \subseteq 3(G)$ and hence $\zeta \in 3(G)$, a contradiction. Thus $[G: \subseteq(\zeta)] \geqq p^{2}$. Clearly $3(G / J) \supseteqq Z(G) / J$. Let $\zeta \in G$ be the inverse image of an element of $3(G / J)$. Then $(G, \zeta) \subseteq J$ 
and $|J|=p$ so $[G: \subseteq(\zeta)] \leqq p$. By the above $\zeta \in \mathbb{Z}(G)$ and the result follows.

We say $p$-group $G$ has property $\left(^{*}\right)$ if $e(G)=e$ and given any $p-e$ nonidentity elements of $G$ there exists an irreducible character $\chi$ of $G$ of degree $p^{e}$ which does not contain any of these elements in its kernel. Note that if $p>e(G)$ and $G$ has property $\left({ }^{*}\right)$, than $G$ is character regular. In [5] we conjectured that every $p$-group satisfies $\left.{ }^{*}\right)$. If this is so the following shows that $p-e$ is best possible.

Proposition 1.4. Given $p$ and $e$. If $p \leqq e$, there exists a $p$-group $G$ with $e(G)=e$ and $\Omega(G)>\langle 1\rangle$. If $p>e$, then there exists a $p$-group $G$ with $e(G)=e$ having $p-e+1$ nonidentity elements with the property that every irreducible character of $G$ of degree $p^{e}$ contains at least one of these elements in its kernel. Moreover in both cases we can take $G$ to have class 2 .

Proof. Let $G$ be generated by $x_{1}, \cdots, x_{e}, y_{1}, \cdots, y_{e}, u, v$ all of order $p$, such that $u$ and $v$ are central, $\left(x_{i}, y_{i}\right)=u v^{i}$ for $i=1, \cdots, e$, and all other commutators are trivial. Set $J=\langle v\rangle$. Clearly $G / J$ is a faithful irreducible linear group of degree $p^{e}$. Since $[G: Z(G)] \leqq p^{2 e}$ we see that $e(G)=e$.

Let $p \leqq e$. We show that $v \in \Omega(G)$. Let $\chi$ be an irreducible character of $G$ with $v \notin \operatorname{ker} \chi$. Then for some $i=1, \cdots, p$ we have $u v^{i} \in \operatorname{ker} \chi$. Since $p \leqq e$ we see that $x_{i}, y_{i}$ exist and that $\bar{x}_{i}$ and $\bar{y}_{i}$ are central in $G /\left\langle u v^{i}\right\rangle$. Hence $G /\left\langle u v^{i}\right\rangle$ has r.x. $(e-1)$ and $\operatorname{deg} \chi \leqq p^{e-1}$. Thus $v \in \Omega(G)$.

Now let $p>e$. Consider the $p-e+1$ elements $v, u v^{e+1}, \cdots, u v^{p}$. Let $\chi$ be an irreducible character of $G$ containing none of these elements in its kernel. Then for some $i=1, \cdots, e$ we have $u v^{i} \in \operatorname{ker} \chi$. As above for such $i \leqq e, G /\left\langle u v^{i}\right\rangle$ has $\mathrm{r} . \mathrm{x} .(e-1)$ and hence the result follows.

We show now that at least in certain cases $(*)$ holds. For possible later applications we use the following general setup.

Let $\mathscr{S}$ be a class of $p$-groups closed under taking subgroups and quotient groups. Let $G$ be a member of $\mathscr{S}$ of minimal order which does not satisfy $\left({ }^{*}\right)$ if such exists. We consider properties of this minimal counterexample.

Let $e(G)=e$ and let $x_{1}, \cdots, x_{r}$ be $r=p-e$ nonidentity elements of $G$ such that each irreducible character of $G$ of degree $p^{e}$ contains at least one of the $x_{i}$ in its kernel. We of course have $r>0$ and thus $p>e$. Clearly $e>0$ by Proposition 4.6 of [2]. Hence $r \leqq p-1$. We can assume that the $x_{i}$ are central and have order $p$ as 
follows. If $x$ is one of the $x_{i}$ 's, then we can find elements $y_{1}, \cdots, y_{k}$ such that $h=\left(x, y_{1}, \cdots, y_{k}\right)$ is a nonidentity central element. If $h \notin \operatorname{ker} \chi$ for some character $\chi$ then clearly $x \notin \operatorname{ker} \chi$. Also we can take a suitable power of $h$ to have order $p$.

We show now that all the $x_{i}$ are contained in $\mathscr{\mathscr { D }}(G)$, the Frattini subgroup of $G$. If not say $x_{1} \notin N$ for some maximal subgroup $N$ of $G$. Since $x_{1}$ is central of order $p$ we have $G=N \times\left\langle x_{1}\right\rangle$ and clearly $e(N)=e(G)=e$. Let $\zeta$ be an element of order $p$ in $3(N)$. Then $\left\langle\zeta, x_{1}\right\rangle$ is central of type $(p, p)$ and has $p+1$ subgroups of order $p$. Since $r \leqq p-1$, we can find one such subgroup $J$ with $x_{1}, \cdots, x_{r}, \zeta \notin J$. Then $G=N \times J$ and $\bar{x}_{1}, \cdots, \bar{x}_{r}$ are nonidentity elements of $G / J \cong N$, a group with $e(G / J)=e$. Since $G$ is a minimal counterexample, we can find a character $\varphi$ of $G / J$ of degree $p^{e}$ with $\bar{x}_{i} \notin \operatorname{ker} \varphi$. Viewing $\varphi$ as a character of $G$ yields a contradiction.

Let $N$ be a maximal subgroup of $G$. Clearly $e(N) \geqq e-1$. If $e(N)=e$, then since $x_{1}, \cdots, x_{r} \in N$ there exists an irreducible character $\varphi$ of $N$ of degree $p^{e}$ with $x_{i} \notin \operatorname{ker} \varphi$ for all $i$. If $\chi$ is a constituent of $\varphi^{*}$ then since $\operatorname{deg} \chi \leqq p^{e}$ we have $\operatorname{deg} \chi=p^{e}$ and $\chi \mid N=\varphi$. Thus $x_{i} \notin \operatorname{ker} \chi$ for all $i$, a contradiction. Therefore $e(N)=e-1$.

If $\chi$ is a character of $G$ of degree $p^{e}$, then $\chi=\varphi^{*}$ for some irreducible character $\varphi$ of $N$ since $e(N)=e-1$. Thus $T(\varphi)=N$ and hence $3(G) \subseteq N$. Therefore $3(G) \subseteq 3(N)$. We show that $3(G)=3(N)$. If not, choose $x_{r+1} \in(G, 3(N))$ with $x_{r+1} \neq 1$. Since $e(N)=e-1$ and $r+1=p-(e-1)$, we can choose an irreducible character $\theta$ of $N$ of degree $p^{e-1}$ with $x_{i} \notin \operatorname{ker} \theta$ for all $i$. Let $\chi$ be a constituent of $\theta^{*}$. If $\operatorname{deg} \chi=p^{e}$, then $x_{1}, \cdots, x_{r} \notin \operatorname{ker} \chi$ yields a contradiction. On the other hand, if $\operatorname{deg} \chi=p^{e-1}$, then $\chi \mid N=\theta$ and so $3(N)$ is central in the representation associated with $\chi$. Hence $(G, 3(N)) \subseteq \operatorname{ker} \chi \cap N=$ $\operatorname{ker} \theta$ and this contradicts the fact that $x_{r+1} \notin \operatorname{ker} \theta$. Thus $3(N)=3(G)$.

We show now that $3(G)$ has two generators and is not cyclic. Let $G$ have as a homomorphic image $\bar{G}=G / K$, a faithful irreducible linear group of degree $p^{e}$. Suppose $3(G) \cap K$ has a subgroup of type $(p, p)$. Then we can find a central subgroup $J$ of order $p$ with $x_{i} \notin J$ for all $i$ and $J \subseteq K$. Then $e(G / J)=e$ and we clearly have a contradiction. Thus $3(G) \cap K$ is cyclic. Since $3(\bar{G})$ is cyclic we see that $3(G)$ has two generators. Let $\zeta \in 3_{2}(G)-3(G)$ with $\zeta^{p} \in \mathbb{Z}(G)$. Then the map $g \rightarrow(g, \zeta)$ is a homomorphism of $G$ into the elements of order $p$ in $3(G)$. The kernel is $\subseteq(\zeta)$ and by the above $[G: \subseteq(\zeta)] \geqq p^{2}$. Hence $[G: \mathbb{C}(\zeta)]=p^{2}$ and $(G, \zeta)$ is abelian of type $(p, p)$. Thus $3(G)$ is not cyclic.

Theorem 1.5. If $G$ has class at most 2 , then $G$ satisfies $\left({ }^{*}\right)$.

Proof. Let $\mathscr{S}$ be the family of all $p$-groups of class at most 2 
and let $G$ be a minimal counterexample. Then all of the above applies. Let $J$ be a central subgroup of $G$ with $x_{i} \notin J$ for all $i$. Let $\chi$ be an irreducible character of $G / J$ viewed as one of $G$ and with $x_{1} J \notin \operatorname{ker} \chi$. Let $K$ be the kernel of $\chi$. If $U$ is the subgroup of $3(G)$ of type $(p, p)$ then clearly $J=K \cap U$ and thus $K \cap 3(G)$ is cyclic. Let $\bar{G}=G / K$. We show that $3(G / K)=3(G) / K$. Let $B$ be the complete inverse image of $3(\bar{G})$ in $G$. Clearly $B \supseteqq 3(G)$. If $B>3(G)$, choose $\zeta \in B-3(G)$ with $\zeta^{p} \in 3(G)$. Since $\bar{\zeta} \in 3(\bar{G})$ we have $(G, \zeta) \subseteq K \cap U=J$. Hence $[G: \Im(\zeta)]=p$, a contradiction. Since $x_{i} \notin \operatorname{ker} \chi$, it follows that $\operatorname{deg} \chi \leqq$ $p^{e-1}$ and so $[\bar{G}: 3(\bar{G})] \leqq p^{2 e-2}$ by Lemma 2.3 of $[2]$. Hence $[G: 3(G)] \leqq$ $p^{2 e-2}$ and $G$ has r.x. $(e-1)$, a contradiction. Thus the theorem is proved.

We now return to our discussion of the general minimal counterexample. Again let $\zeta \in 3_{2}(G)-3(G)$ with $\zeta^{p} \in 3(G)$. Thus if $K=(5(\zeta)$, then we have $[G: K]=p^{2}$ and in fact $G / K \cong(G, \zeta)$ is abelian of type $(p, p)$. Let $N$ be any subgroup of $G$ with $G>N>K$. Since $3(K) \nsubseteq$ $3(N)$ and $K$ is character regular we see by Proposition 1.3 (i) that $\epsilon(K)<e(N)$. But $\epsilon(K) \geqq e(N)-1$ so $e(K)=\epsilon(N)-1=e-2$. In rarticular $e \geqq 2$.

We show now that $[3(K): 3(G)]=p$ so that $3(\mathfrak{E}(\zeta))=\langle 3(G), \zeta\rangle$. Let $\theta$ be an irreducible character of $K$ of degree $p^{e-2}$ (note that $e(K)=$ $e-2)$ with $x_{1}, \cdots, x_{r}$ not in its kernel and let $J \subseteq \operatorname{ker} \theta$ where $J$ is central in $G$ of order $p$. Clearly $J=(T, \zeta)$ for some subgroup $T$ with $G>T>K$. Consider $\bar{G}=G / J$. Since $\bar{x}_{i} \neq 1$ in $\bar{G}$ we see that $e(\bar{G}) \leqq e-1$. But $e(\bar{K})=e-2$, where of course $\bar{K}=K / J$. Also $\bar{\zeta} \in 3(\bar{T})-3(\bar{G})$. Hence $e(\bar{G})>e(\bar{T}) \geqq e(\bar{K})$. This yields $e(\bar{G})=e-1$ and $e(\bar{T})=e(\bar{K})=e-2$. By Proposition 1.3 (i) we have $\overline{3(K)} \subseteq$ $3(\bar{K}) \subseteq 3(\bar{T})$ and thus $(T, 3(K))=J$. Now $T=\langle K, a\rangle$ and the map $b \rightarrow(b, a)$ is a homomorphism of $3(K)$ onto $J$ with kernel $\subseteq(a) \cap 3(K)=$ $3(T)$. Hence $[3(K): 3(T)]=p$. But $3(T)=3(G)$ so $[3(K): 3(G)]=p$. If $e=2$, then $K$ is abelian and so $3(K)=K$. Hence $[G: 3(G)]=p^{3}$, a contradiction and hence $e \geqq 3$. If we let $\mathscr{S}$ be the set of $p$-groups with r.x.2, then the above yields:

Proposition 1.6. If $G$ is a $p$-group with $\mathrm{r} . \mathrm{x} .2$, then $G$ has property $(*)$.

We now discuss an application of the above. Let $\mathscr{T}$ denote a family of character regular $p$-grours closed under taking subgroups and quotient groups.

Proposition 1.7. Let $G \in \mathscr{T}$ with $e(G)=e$. Let $\chi$ be an irreducible character of $G$ of degree $p^{e}$ and let $Z_{\chi}$ denote the set of 
elements of $G$ central in the representation associated with $\chi$. Then $Z_{\chi}$ is abelian.

Proof. If $Z_{x}$ is central the result is clear. So assume $Z_{x} \nsubseteq 3(G)$ and hence $Z_{\chi}>3(G)$. Choose $\zeta \in Z_{\chi}-3(G)$ with $\zeta \in B_{2}(G)$ and $\zeta^{p} \in 3(G)$. Then $(\zeta, G)$ is central, elementary abelian and $(\zeta, G) \subseteq$ ker $\chi$. Clearly $(\zeta, G) \neq\langle 1\rangle$. If $|(\zeta, G)| \geqq p^{2}$, choose $J_{1}$ and $J_{2}$ subgroups of $(\zeta, G)$ of order $p$ with $J_{1} \cap J_{2}=\langle 1\rangle$. Since $J_{i} \subseteq \operatorname{ker} \chi$, we have $e\left(G / J_{i}\right)=e$ and hence by induction $Z_{\chi} / J_{i}$ is abelian. Thus $Z_{\chi}^{\prime} \cong J_{1} \cap J_{2}=\langle 1\rangle$ and $Z_{\chi}$ is abelian.

Thus we can assume that $(\zeta, G)=p$ and hence if $H=(\zeta)(\zeta)$, then $[G: H]=p$. Since $H$ is character regular and $3(H) \nsubseteq 3(G)$, Proposition 1.3 (i) yields $e(H)=e-1$. Thus $\chi \mid H=\sum_{1}^{p} \varphi_{i}$ and $\chi$ vanishes off $H$. This latter fact implies that $Z_{\chi} \subseteq H$. Now if $\varphi=\varphi_{1}$, then $\operatorname{deg} \varphi=p^{e-1}$ and $e(H)=e-1$. Thus in $H, Z_{\varphi}$ is abelian. Since clearly $Z_{\chi} \subseteq Z_{\varphi}$, the result follows.

Corollary 1.8. Let $G$ have class 2. If $p>e(G)=e$, then $G$ has a normal abelian subgroup of index $p^{2 e}$.

Proof. Let $\mathscr{T}$ be the family of $p$-groups of class $\leqq 2$ with $p>e(G)$. By Theorem 1.5 all members of $\mathscr{T}$ are character regular. Let $\chi$ be an irreducible character of $G$ of degree $p^{e}$. Then by the above $Z_{\chi}$ is a normal abelian subgroup of $G$. Since $G$ has class $2,\left[G: Z_{\chi}\right]=p^{2 e}$ and the result follows.

2. $\pi$-Character groups. In this section we study groups whose irreducible characters have degrees which are powers of a fixed integer $m$. In fact we consider the more general class of groups defined below. Here $\pi(n)$ denotes the set of prime factors of integer $n$.

DEFINITION 2.1. Let $\pi$ be a set of primes. We say group $G$ is a $\pi$-character group if the following hold.

(i) The distinct degrees of the irreducible characters of $G$ are $d_{0}, d_{1}, \cdots, d_{k}$ with $k \geqq 1$.

(ii) For all $i \geqq 1, d_{i-1} \mid d_{i}$ and $\pi\left(d_{i}\right)=\pi$.

(iii) If $\pi=\{p\}$, then $\mathfrak{S}_{p}(G)$ is abelian.

Condition (iii) above is included for convenience in order to avoid overlap with our previous study of r.x. $e$ groups. If $H$ is a homomorphic image of $G$, then the degrees of the irreducible characters of $H$ forms subset of those of $G$. Hence if $G$ is a $\pi$-character group, then either $H$ is a $\pi$-character group or $H$ is abelian. The main result here is as follows. 
THEOREM 2.2. Let $G$ be a $\pi$-character group. Suppose the distinct degrees of its irreducible characters are $d_{0}, d_{1}, \cdots, d_{k}$ with $d_{i-1} \mid d_{i}$. Then $G$ has the following structure.

(i) $G$ has a normal abelian $\mathfrak{S}_{\pi^{\prime}}$ subgroup $H \neq\langle 1\rangle$ with $G / H \cong$ $\mathfrak{S}_{\pi}(G)$ abelian.

(ii) $A=\sqrt{ }(H)$ is a normal abelian subgroup of $G$ of index $d_{k}$.

(iii) There exists a subset $\left\{a_{0}, a_{1}, \cdots, a_{r}\right\}$ of $\{0,1, \cdots, k\}$ with $0=a_{0}<a_{1}<\cdots<a_{r}=k$ such that $G / A$ is abelian of type $\left(d_{a_{1}} / d_{a_{0}}\right.$, $\left.d_{a_{2}} / d_{a_{1}}, \cdots, d_{a_{r}} / d_{a_{r-1}}\right)$ and $\left(d_{a_{i+1}} / d_{a_{i}}\right) \mid\left(d_{a_{i}} / d_{a_{i-1}}\right)$ for all $i$.

CoRollary 2.3. Suppose the degrees of the irreducible characters of $G$ are all powers of a fixed integer $m$, with $m^{s}$ the largest such degree. Let $\pi=\pi(m)$ and assume that $|\pi|>1$. Then $G$ has a normal abelian subgroup $A$ with $G / A$ abelian of order $m^{s}$ and type $\left(m^{s_{0}}, m^{s_{1}}\right.$, $\cdots, m^{S}$ ) for suitable integers $s_{i}$. Moreover $\mathfrak{S}_{\pi}(G)$ is abelian.

The corollary is of course an immediate consequence of the theorem. The proof of the latter will be in two parts. We first show that $G$ satisfies (i). Then we study groups with that property and show that they satisfy the remaining conditions (ii) and (iii).

We start with a lemma. If $\lambda$ is a linear character of $G$, then the order of $\lambda$, written $o(\lambda)$, is its order as an element of the dual group $\widehat{G / G^{\prime}}$. If $\chi$ is any irreducible character of $G$ we set $o(\chi)$ equal to $o(\lambda)$ where $\lambda=\operatorname{det} \chi$, the determinant of the representation associated with $\chi$.

Lemma 2.4. Let $p$ be a prime and let $U=\mathfrak{H}_{p}(G)$ be the minimal normal subgroup of $G$ having a p-quotient group. Then

$$
|U| \equiv \sum_{p \nmid o(x)} \chi(1)^{2} \quad \bmod p .
$$

Proof. By induction on $|G|$. Suppose first that $G$ has no normal subgroup of index $p$. Then $G=\mathfrak{U}_{p}(G)$ and $G / G^{\prime}$ is a $p^{\prime}$-group. Hence for all $\lambda \in \widehat{G / G^{\prime}}$ we have $p \nmid o(\lambda)$. Therefore the above congruence follows from the equation $|G|=\sum \chi(1)^{2}$.

Now let $G$ have a normal subgroup $H$ of index $p$. Clearly $\mathfrak{u}_{p}(G)=$ $\mathfrak{u}_{p}(H)$ and thus by induction

$$
|U| \equiv \sum_{p \nmid o(\chi)} \theta(1)^{2} \quad \bmod p
$$

where the sum runs over the irreducible characters $\theta$ of $H$. We show now that

$$
\sum_{p \nmid o(\chi)} \chi(1)^{2} \equiv \sum_{p \nmid o(\theta)} \theta(1)^{2} \quad \bmod p .
$$

In both sums we can of course discard those $\chi$ and $\theta$ with $p \mid \chi(1)$ and 
$p \mid \theta(1)$. Also if $T(\theta)=H$, then $\theta$ has $p$ conjugates $\theta_{1}, \theta_{2}, \cdots, \theta_{\zeta}$. Clearly $\theta_{i}(1)=\theta(1)$ and $o\left(\theta_{i}\right)=o(\theta)$. Thus the contribution of these conjugates to the right hand sum is a multiple of $p$. Hence we need only consider those $\theta$ with $T(\theta)=G$.

Let $\mathscr{S}_{1}=\{\chi \mid \chi$ is an irreducible character of $G, p \nmid \chi(1)$, and $p \nmid o(\chi)\}$ and $\mathscr{S}_{2}=\{\theta \mid \theta$ is an irreducible character of $H, p \nmid \theta(1)$, $p \nmid o(\theta)$, and $T(\theta)=G\}$. As we have shown above it suffices to prove that

$$
\sum_{\mathscr{S}_{1}} \chi(1)^{2} \equiv \sum_{\mathscr{\sigma}_{2}} \theta(1)^{2} \quad \bmod p
$$

We will in fact show that the map $\chi \rightarrow \chi \mid H$ is a one-to-one map of $\mathscr{S}_{1}$ onto $\mathscr{S}_{2}$ and this will yield the result since $\chi$ and $\chi \mid H$ have the same degree.

Let $\chi \in \mathscr{S}_{1}$. Since $[G: H]=p$ we have that either $\chi \mid H$ is irreducible or $\chi \mid H$ is the sum of $p$ conjugates. Since $p \nmid \chi(1)$, the latter cannot occur so $\chi \mid H=\theta$ is irreducible. Clearly $\theta(1)=\chi(1)$, $o(\theta) \mid o(\chi)$ and $T(\theta)=G$ and hence $\theta \in \mathscr{S}_{2}$. Thus the restriction map sends $\mathscr{S}_{1}$ into $\mathscr{S}_{2}$.

Now let $\theta \in \mathscr{S}_{2}$ and let $\mu=\operatorname{det} \theta$. Since $T(\theta)=G$ we have $T(\mu)=G$ and thus $K=\operatorname{ker} \mu$ is normal in $G$. If $\chi$ is such that $\chi \mid H=\theta$, then $\chi$ is a constituent of $\theta^{*}$. Thus to show that the restriction map is one-to-one and onto we must find a unique constituent $\chi$ of $\theta^{*}$ with $\chi \in \mathscr{S}_{1}$ and $\chi \mid H=\theta$. Let $\tau$ be a nonprincipal linear character of $G / H$ and let $\chi$ be an irreducible constituent of $\theta^{*}$. Since $[G: H]=p$ and $T(\theta)=G$ we see that $\chi \mid H=\theta$ and that all the constituents of $\theta^{*}$ are of the form $\chi_{i}=\tau^{i} \chi$ for $i=0,1, \cdots, p-1$. Let $\lambda=\operatorname{det} \chi$ so that $\lambda \mid H=\mu$. We have clearly

$$
\operatorname{det} \chi_{i}=\operatorname{det} \tau^{i} \chi=\tau^{i \times(1)} \lambda .
$$

Since $\chi(1)=\theta(1)$ is prime to $p$ we see that $\operatorname{det} \chi_{i} \neq \operatorname{det} \chi_{j}$ for $i \neq j$ and hence we obtain $p$ distinct linear characters of $G$ which extend $\mu$.

Since $T(\mu)=G$ we see that $H / K$ is central in $G / K$ and since $G / H$ is cyclic, $G / K$ is abelian. Also $H / K$ is a $p^{\prime}$-group since $p \nmid o(\mu)$ and hence $G / K \simeq(H / K) \times(G / H)$. It follows easily from this that there are precisely $p$ distinct linear characters of $G$ which extend $\mu$ and that precisely one of these has order prime to $p$. Hence there is a unique $i_{0}$ with $o\left(\chi_{i_{0}}\right)$ prime to $p$. Then $p \nmid o\left(\chi_{i_{0}}\right)$ and $p \nmid \chi_{i_{0}}(1)$ since $\chi_{i_{0}}(1)=$ $\theta(1)$. Thus $\chi_{i_{0}} \in \mathscr{S}_{1}$ and $\chi_{i_{0}} \mid H=\theta$. This completes the proof.

The first two parts of the following theorem are due to John Thompson. They generalize our original result, proved under more restrictive assumptions.

THEOREM 2.5. Let $p$ be a prime and $\pi$ a set of primes. 
(i) Suppose for every nonlinear irreducible character $\chi$ of $G$ we have $p \mid \chi(1)$. Then $G$ has a normal p-complement.

(ii) If the degrees of the irreducible characters of $G$ are linearly ordered by divisibility, then $G$ has a Sylow tower.

(iii) Suppose for every nonlinear irreducible charactor $\chi$ of $G$ we have $\pi(\chi(1))=\pi$. Then $G$ has a normal abelian $\mathfrak{S}_{\pi^{\prime}}$ subgroup $H$. Moreover if $|\pi|>1$, then $G / H$ is abelian.

Proof. (i) Let $U$ be as in the preceeding lemma. Since $p \mid \chi(1)$ if $\chi(1) \neq 1$ we see by Lemma 2.4 that

$$
|U| \equiv \sum_{p \nmid o(\lambda)} \lambda(1)^{2} \quad \bmod p
$$

where the sum runs over linear characters $\lambda$. Clearly $p \nmid o(\lambda)$ is equivalent to $\lambda$ belonging to $\mathfrak{S}_{p^{\prime}}\left(\widehat{G / G^{\prime}}\right)$. Hence

$$
|U| \equiv\left|\Im_{p^{\prime}}\left(\widehat{G / G^{\prime}}\right)\right| \quad \bmod p
$$

and so $p \nmid|U|$. Thus $U$ is a normal $p$-complement of $G$.

(ii) By induction on $|G|$. If $G$ is abelian the result is clear so assume that $G$ is nonabelian. Let $d_{0}=1, d_{1}, \cdots, d_{k}$ be the distinct degrees of the irreducible characters of $G$ with $d_{i} \mid d_{i+1}$. Since $k \geqq 1$, choose prime $p$ with $p \mid d_{1}$. Then for all $i \geqq 1, p \mid d_{i}$. By (i), $G$ has a normal $p$-complement $H$. Let $\chi$ be a character of $G$ of degree $d_{i}$ and say

$$
\chi \mid H=a \sum_{1}^{t} \theta_{i} .
$$

If $\theta=\theta_{1}$, then at $\operatorname{deg} \theta=\operatorname{deg} \chi=d_{i}$. As is well known at $\mid[G: H]$ and of course $\operatorname{deg} \theta|| H \mid$. Hence clearly $a t=\left|d_{i}\right|_{p}$ and $\operatorname{deg} \theta=\left|d_{i}\right|_{p^{\prime}}$. Thus the degrees of the irreducible characters of $H$ are $\left|d_{0}\right|_{p^{\prime}},\left|d_{1}\right|_{p^{\prime}} \mid, \cdots$, $\left|d_{k}\right|_{p^{\prime}}$ and these are linearly ordered by divisibility. By induction $H$ has a Sylow tower and thus the result follows here.

(iii) By (i), $G$ has a normal $p$-complement for all $p \in \pi$. Hence $G$ has a normal $\mathfrak{S}_{\pi^{\prime}}$ subgroup $H$ with $G / H$ nilpotent. Let $\theta$ be an irreducible character of $H$ and let $\chi$ be a constituent of $\theta^{*}$. Then $\operatorname{deg} \theta \mid \operatorname{deg} \chi$ and $\operatorname{deg} \theta|| H \mid$ and so $\operatorname{deg} \theta=1$. Thus $H$ is abelian. Now let $\pi=\left\{p_{1}, p_{2}, \cdots, p_{r}\right\}$ and suppose $r>1$. Let $G / H=P_{1} \times P_{2} \times \cdots \times P_{r}$ where $P_{i}=\mathfrak{S}_{p_{i}}(G / H)$. If $P_{i}$ is nonabelian then $G / H$ has a character $\chi$ with $\pi(\chi(1))=\left\{p_{i}\right\} \neq \pi$, a contradiction. Hence for all $i, P_{i}$ is abelian and thus $G / H$ is abelian. This completes the proof.

Part (iii) of the above result yields (i) of Theorem 2.2. We now study groups satisfying this latter condition.

THeOREM 2.6. Let $\pi$ be a set of primes. Let $G$ be a group with a normal abelian $\mathfrak{S}_{\pi^{\prime}}$ subgroup $H$ and with $G / H \cong \mathfrak{S}_{\pi}(G)$ abelian. 
Suppose the distinct degrees of the irreducible characters of $G$ are $d_{0}, d_{1}, \cdots, d_{k}$ with $d_{i-1} \mid d_{i}$. Then

(i) $A=\sqrt{ }(H)$ is a normal abelian subgroup of $G$.

(ii) There exists a subset $\left\{a_{0}, a_{1}, \cdots, a_{r}\right\}$ of $\{0,1, \cdots, k\}$ with $0=a_{0}<a_{1}<\cdots<a_{r}=k$ such that $G / A$ is abelian of type $\left(d_{a_{1}} / d_{a_{0}}\right.$, $\left.d_{a_{2}} / d_{a_{1}}, \cdots, d_{a_{r}} / d_{a_{r-1}}\right)$ so $[G: A]=d_{k}$ and $\left(d_{a_{i+1}} / d_{a_{i}}\right) \mid\left(d_{a_{i}} / d_{a_{i-1}}\right)$ for all $i$.

Let $K$ be a normal subgroup of $G$, maximal subject to $G / K$ being nonabelian. If $G / K=E$ is solvable, then in the terminology of $\S 2$ of [2], $E$ is extra-special. By Proposition 2.2 of [2], $E$ is either a Case $P$ or Case $Q$ group. We will refer to these as Case $P$ and Case $Q$ quotients of $G$.

Let $G$ satisfy the hypotheses of the above theorem. Set $q_{i}=$ $d_{i} / d_{i-1}$. These degree quotients will come into play in some later results.

Lemma 2.7. Let $G$ satisfy the hypothesis of Theorem 2.6. Let $K \triangle G$ so that $G / K$ is an extra-special group. Then $G / K$ is a Case $Q$ quotient. Let $Q / K$ be the normal Sylow $q$-subgroup of $G / K$ with $G / Q$ cyclic of order $d$. Then $|d|_{\pi}=d$ and $|q|_{\pi^{\prime}}=q$. Also there exists $a$ subset $\left\{b_{0}, b_{1}, \cdots, b_{s}\right\}$ of $\{0,1, \cdots, k\}$ with $b_{0}<b_{1}<\cdots<b_{s}=k$ such that the distinct degrees of the irreducible characters of $Q$ are $d_{b_{0}} / d$, $d_{b_{1}} / d, \cdots, d_{b_{s}} / d$.

Suppose further that $q_{i} \nmid d$ for all $i>1$. Then $d=d_{1}$ and the distinct degrees of the irreducible characters of $Q$ are $d_{1} / d_{1}, d_{2} / d_{1}, \cdots$, $d_{k} / d_{1}$. Moreover if $\theta$ is an irreducible character of $Q$, then $\theta^{*}$ is either irreducible or it has all linear constituents.

Proof. Let $G / K$ be an extra-special quotient of $G$. If $G / K$ is Case $P$, then $G / K$ is a nonabelian $p$-group for some prime $p$. Since all Sylow subgroups of $G$ are abelian, this cannot occur. Thus $G / K$ is Case $Q$. By Ito's theorem we have $d_{i} \mid[G: H]$ for all $i$ and hence $\left|d_{i}\right|_{\pi}=d_{i}$. Since $d$ is the degree of an irreducible character of $G$ we have $|d|_{\pi}=d$. Moreover since $G / K$ is nonabelian and $\mathfrak{S}_{\pi}(G)$ is abelian, we see that $G / K$ is not a $\pi$-group. Hence $|q|_{\pi^{\prime}}=q$.

Let $\theta$ be an irreducible character of $Q$ and let $\mu$ be a nonprincipal linear character of $Q / K$ viewed as one of $Q$. Suppose $\theta=\theta \mu$. If $L$ is the kernel of $\mu$ then $Q>L \supseteqq K$ and $\theta$ vanishes off $L$. Say $\theta \mid L=$ $a \sum_{1}^{t} \varphi_{i}$. Then $[\theta|L, \theta| L]_{L}=a^{2} t$. On the other hand since $\theta$ vanishes off $L,[\theta|L, \theta| L]_{L}=[Q: L][\theta, \theta]=[Q: L]$. Hence $a^{2} t$ is a proper power of $q$. Since $\operatorname{deg} \theta=a t \operatorname{deg} \varphi_{1}$ we have $q \mid \operatorname{deg} \theta$. If $\chi$ is a constituent of $\theta^{*}$, then $\operatorname{deg} \theta \mid \operatorname{deg} \chi$ and so $q \mid \operatorname{deg} \chi$. This is a contradiction since $|\operatorname{deg} \chi|_{\pi}=\operatorname{deg} \chi$ and $q \notin \pi$. Hence $\theta \neq \theta \mu$.

Now let $\lambda, \mu$ be two distinct characters of $Q / K$. We show that 
$T(\theta \lambda) \cap T(\theta \mu)=Q$. If not we can find $x \in(T(\theta \lambda) \cap T(\theta \mu))-Q$. Then

$$
\theta \lambda=(\theta \lambda)^{x}=\theta^{x} \lambda^{x} \quad \theta \mu=(\theta \mu)^{x}=\theta^{x} \mu^{x}
$$

and hence

$$
\theta^{x}=\theta \lambda \bar{\lambda}^{x}=\theta \mu \bar{\mu}^{x}
$$

Now $\lambda \bar{\lambda}^{x} \neq \mu \bar{\mu}^{x}$ since $x$ acts fixed point free on $Q / K$ and $\lambda \neq \mu$. Thus $\theta=\theta \rho$ where $\rho=\left(\mu \bar{\mu}^{x}\right)\left(\bar{\lambda} \lambda^{x}\right) \neq 1$ and this contradicts the above. Let $u$ be the number of minimal subgroups of $G / Q$. Then this says that there are at most $u$ characters $\lambda$ of $Q / K$ with $T(\theta \lambda)>Q$. Clearly $u \leqq d-1$ since each minimal subgroup is cyclic and has a nonidentity generator. On the other hand $G / Q$ acts fixed point free on $Q / K$ so there are at least $d+1>u$ linear characters of $Q / K$. Hence there exists $\lambda$ with $T(\theta \lambda)=Q$.

Since $T(\theta \lambda)=Q$, it follows easily that $(\theta \lambda)^{*}$ is irreducible. Hence for some $i$

$$
d_{i}=\operatorname{deg}(\theta \lambda)^{*}=d \operatorname{deg}(\theta \lambda)=d \operatorname{deg} \theta .
$$

This implies that there exists a subset $\left\{b_{0}, b_{1}, \cdots, b_{s}\right\}$ of $\{0,1, \cdots, k\}$ with $b_{0}<b_{1}<\cdots<b_{s} \leqq k$ such that the distinct degrees of the irreducible characters of $Q$ are $d_{b 0} / d, d_{b_{1}} / d, \cdots, d_{b_{s}} / d$. We show now that $b_{s}=k$. Let $\chi$ be a character of $G$ of degree $d_{k}$ and let $\theta$ be an irreducible constituent of $\chi \mid Q$. Then certainly $\operatorname{deg} \theta \geqq d_{k} / d$. On the other hand by the above $\operatorname{deg} \theta=d_{j} / d$ for some $j$. Hence $d_{j} / d \geqq d_{k} / d$ so $j=k$ and $\operatorname{deg} \theta=d_{k} / d$. This completes the first half of the proof.

Now assume that $q_{i} \nmid d$ for all $i>1$. Since $q_{i} \mid d_{i}$ and $d>1$, it follows that $d=d_{1}$. Let $\chi$ be an irreducible character of $G$ of degree $d_{i}$ for $i>0$ and let $\theta=\theta_{1}$ be an irreducible constituent of $\chi \mid Q$. We have $\chi \mid Q=a \sum_{1}^{t} \theta_{i}$ and thus if $b=a t$ then $b \leqq d$ and $b \operatorname{deg} \theta=\operatorname{deg} \chi=$ $d_{i}$. On the other hand we know that $\operatorname{deg} \theta=d_{j} / d$ for some $j$. Hence $d_{i} / b=d_{j} / d$. Since $d \geqq b$, it follows that $d_{j} \geqq d_{i}$. If $d_{j}>d_{i}$, then $d_{i+1} \mid d_{j}$ and we have

$$
d=d_{1}=b\left(d_{j} / d_{i}\right)=b q_{i+1}\left(d_{j} / d_{i+1}\right)
$$

and $q_{i+1} \mid d$, a contradiction for $i>0$. Hence $i=j$ and $\operatorname{deg} \theta=d_{i} / d$. Moreover $b=a t=d$ and since $a^{2} t \leqq d$, in general, we have $a=1$, $t=d$ and $\chi=\theta^{*}$. Thus the distinct degrees of the irreducible characters of $Q$ are $d_{1} / d_{1}, d_{2} / d_{1}, \cdots, d_{k} / d_{1}$.

Finally let $\theta$ be a character of $Q$ and suppose that $\theta^{*}$ has a nonlinear irreducible constituent $\chi$. Since $\theta$ is a constituent of $\chi \mid Q$, the above yields $\chi=\theta^{*}$ is irreducible. This completes the proof of the lemma. 
Proof of Theorem 2.6. First $A=\mathfrak{S}_{\pi}(A) H, \mathfrak{S}_{\pi}(A)$ is abelian and $H$ is central in $A$. Hence $A$ is abelian and (i) follows. Note that $G$ is solvable. If $G$ is abelian, then (ii) is obvious. So assume $G$ is nonabelian.

Let $K \triangle G$ with $G / K$ an extra-special group. By the preceeding lemma, $G / K$ is a Case $Q$ quotient. Using the notation of that lemma we have $[G: Q]=d_{i}$ for some $i$. Moreover assume that $K$ is so chosen that $i$ is maximal with this occuring.

In $G / K$ we have $\mathfrak{夭}_{G / K}(Q / K)=Q / K$. This shows that $A=\mathbb{C}_{G}(H) \subseteq Q$ and hence $\mathfrak{r}_{G}(H)=\mathfrak{V}_{Q}(H)=A$. Let $x \in G / A$ be such that it generates the cyclic quotient $G / Q$. We show that $|\langle x\rangle|=d_{i}=d_{i} / d_{0}$. Clearly $d_{i}||\langle x\rangle \mid$. If $d_{i} \neq|\langle x\rangle|$, then for some prime $p \in \pi$ we have $\left.|\langle x\rangle|_{p}\right\rangle$ $\left|d_{i}\right|_{p}$. For this prime let $J$ be the subgroup of $\langle x\rangle$ of order $p$. Now $\mathfrak{S}_{\pi}(A)$ centralizes $H$ and some $\mathfrak{\Im}_{\pi}(G)$ and hence $\mathfrak{S}_{\pi}(A)$ is central in $G$. Thus by Lemma 1.2 we can write $A=D \times C$ where $D=\mathbb{E}_{A}(J)$ and $D, C \triangle G$ and $J$ acts fixed point free on $C$. Clearly $C \neq\langle 1\rangle$. Let $\lambda$ be a nonprincipal linear character of $C$ viewed as one of $A$. Then $(T(\lambda) / A) \cap J=\langle 1\rangle$ and hence $\left.[G: T(\lambda)]_{p} \geqq|\langle x\rangle|_{p}\right\rangle\left|d_{i}\right|_{p}$. Since $C \neq 1$ this implies that the distinct degrees of the irreducible characters of $G / D$ are $1, d_{j}, \cdots$ with $j>i$. Hence $G / D$ has a Case $Q$ quotient with $[G: Q]=d_{s}>d_{i}$, a contradiction. Thus $|\langle x\rangle|=d_{i}$. Setting $a_{1}=i$, we have by induction applied to $Q$, that $G / A$ is abelian of type $\left(d_{a_{1}} / d_{a_{0}}\right.$, $\left.d_{a_{2}} / d_{a_{1}}, \cdots, d_{a_{r}} / d_{a_{r-1}}\right)$ with $a_{r}=k$. Also $\left(d_{a_{j+1}} / d_{a_{j}}\right) \mid\left(d_{a_{j}} / d_{a_{j-1}}\right)$ for $j>1$ by induction. To obtain $\left(d_{a_{2}} / d_{a_{1}}\right) \mid\left(d_{a_{1}} / d_{a_{0}}\right)$ we merely note that $|\langle x\rangle|=d_{i}$ for all such choices of $x$. This implies that the period of $Q / A$ divides $d_{i}=d_{a_{1}} / d_{a_{0}}$. This completes the proof.

The proof of Theorem 2.2 is now immediate. Part (i) follows from Theorem 2.5 (iii) and from the assumption that if $\pi=\{p\}$, then $\mathfrak{S}_{p}(G)$ is abelian. Then Theorem 2.6 yields parts (ii) and (iii).

In the remainder of this section we assume that $G$ satisfies the hypothesis of Theorem 2.6 and we will use the notation of the conclusion of that theorem. We first note a few simple facts about the characters of $G$.

Lemma 2.8. Let $\chi$ be an irreducible character of $G$. Then we have

(i) $\chi \mid H=\sum_{1}^{t} \lambda_{i}$, that is there is no ramification.

(ii) There exists a subgroup $L \supseteqq A$ and a linear character $\lambda$ of $L$ with $\chi=\lambda^{*}$.

(iii) If $\chi$ is faithful, then $L=A$ and $\operatorname{deg} \chi=d_{k}$.

Proof. Let $\chi \mid H=a \sum_{1}^{t} \lambda_{i}$ and set $L=T\left(\lambda_{1}\right)$. Clearly $L \supseteqq \Im(H)=$ 
A. As is well known there exists a character $\theta$ of $L$ with $\chi=\theta^{*}$ and $\theta \mid H=a \lambda_{1}$. Let $K$ be the kernel of $\theta$. Then clearly $H$ is central modulo $K$. Since $\widetilde{S}_{-}(L)$ is abelian this shows that $L / K$ is abelian and hence $\operatorname{deg} \theta=1$. Thus $a=1$ and (i) and (ii) are proved.

If $\chi$ is faithful then since $L \triangle G$ we have that $L$ is abelian. Hence $L \subseteq \subseteq(H)=A$. This yields $\operatorname{deg} \mathcal{X}=[G: A]=d_{k}$ and (iii) follows.

It is interesting to consider which subgrours $L$ can occur in (ii) of the above lemma. Define a Galois connectivity between groups $L$ with $G \supseteqq L \supseteqq A$ and groups $B$ with $H \supseteqq B$ as follows.

$$
L \stackrel{d}{\longrightarrow}(L, H) \quad B \stackrel{u}{\longrightarrow}\{g \in G \mid(g, H) \cong B\}
$$

We say $L$ is closed if $L^{d u}=L$

Proposition 2.9. Using the above notation, group $L$ has a linear character $\lambda$ with $\lambda^{*}$ irreducible if and only if $L$ is closed.

Proof. We note first that $(L, H)=L^{\prime}$. This follows since $L /(L, H)$ has a central $\Im_{\pi^{\prime}}$ subgroup and an abelian $\subseteq_{\pi}$ subgroup and hence is abelian.

Now let $L$ have a linear character $\lambda$ with $\lambda^{*}=\chi$ irreducible. Set $M=L^{d u}$ so that $M \supseteqq L$. Suppose that $M>L$. Clearly $L \supseteqq \operatorname{ker} \chi$. Since $G / A$ is abelian, $L \triangle G$ and hence $L / \operatorname{ker} \chi$ is abelian. Thus $L^{\prime}=$ $(L, H) \cong \operatorname{ker} \chi$. Since $\operatorname{deg} \chi=[G: L]$ and $M>L$ it follows that $M^{\prime}=(M, H) \nsubseteq \operatorname{ker} \chi$. Thus $M^{d}=(M, H) \neq(L, H)=L^{d}$, a contradiction. Hence $M=L$ and $L$ is closed.

Now assume $L$ is closed. We consider $\bar{G}=G /(L, H)$ in which $\bar{L}=L /(L, H)$ is abelian. Since $L$ is closed we see that $\bar{G} / \bar{L}$ acts faithfully on $\bar{D}=\Im_{\pi^{\prime}}(\bar{L})$. Thus $\bar{G} / \bar{L}$ acts faithfully on $\hat{\bar{D}}$, the dual group of $\bar{D}$. Since these groups are abelian and have relatively prime orders, it follows by a trivial modification of Lemma 2.2 of [5] that there exists $\lambda \in \hat{\bar{D}}$ with $\widetilde{S}_{\vec{G} / \bar{L}}(\lambda)=\langle 1\rangle$. View $\lambda$ as a character of $\bar{L}$ and then as one of $L$. We see that $T(\lambda)=L$ and hence that $\lambda^{*}$ is irreducible. The result follows.

If $G / A$ is cyclic we can obtain additional information.

THEOREM 2.10. Suppose $G / A$ is cyclic. Let $L_{i}$ be the unique subgroup of $G$ with $\left[G: L_{1}\right]=d_{i}$ and $L_{i} \supseteqq A$. Then we can write $A=$ $B_{0} \times B_{1} \times \cdots \times B_{k}$ where each $B_{1}$ is characteristic in $G, L_{i}$ centralizes $B_{i}$ and $G / L_{i}$ acts fixed point free on $B_{i}$. Here $B_{i} \neq\langle 1\rangle$ for $i \neq 0$. Conversely a group with this structure has characters of degrees $d_{0}, d_{1}, \cdots, d_{k}$ only.

Proof. Note that $A=\subseteq(H)$ and each $L_{i}$ is characteristic in $G$. 
Note also that $\mathfrak{S}_{\pi}(A)$ is central in $G$. We have

$$
\langle 1\rangle \subseteq \mathfrak{C}_{A}\left(L_{0}\right) \subseteq \mathfrak{C}_{A}\left(L_{1}\right) \subseteq \cdots \subseteq \mathfrak{V}_{A}\left(L_{k}\right)=A
$$

and each of these groups is characteristic in $G$. By Lemma 1.2 we can write for $i=1,2, \cdots, k$

$$
\mathfrak{V}_{A}\left(L_{i}\right)=\mathfrak{V}_{A}\left(L_{i-1}\right) \times B_{i}
$$

where each $B_{i}$ is characteristic in $G$. Setting $B_{0}=\mathfrak{C}_{A}\left(L_{0}\right)=3(G)$ we have

$$
A=B_{0} \times B_{1} \times \cdots \times B_{k}
$$

where each $B_{i}$ is characteristic in $G$ and is centralized by $L_{i}$.

Let $i \geqq 1$ and let $\lambda$ be a nonprincipal linear character of $B_{i}$ viewed as one of $A$. Since $L_{i}$ centralizes $B_{i}$ we have $T(\lambda) \supseteqq L_{i}$. If $T(\lambda)>L_{i}$, then by Lemma 2.8 (i) we have $T(\lambda) \supseteqq L_{i-1}$. This implies that $L_{i-1}$ centralizes an element of $B_{i}^{\#}$ which is not the case by definition of $B_{i}$. Hence $T(\lambda)=L_{i}$ and $G / L_{i}$ acts fixed point free on $\hat{B}_{i}$ and hence on $B_{i}$.

We show now that $B_{i} \neq\langle 1\rangle$ for $i \neq 0$. Let $\chi$ be an irreducible character of $G$ of degree $d_{i}$ and let $\lambda$ be an constituent of $\chi \mid A$. Since there is no ramification, $[G: T(\lambda)]=d_{i}$ and hence $T(\lambda)=L_{i}$. Write $\lambda=\lambda_{0} \lambda_{1} \cdots \lambda_{k}$ where $\lambda_{j}$ is a character of $B_{j}$ viewed as one of $A$. As we showed above, $L_{i}$ fixes no nonprincipal character of $B_{j}$ for $j>i$. Hence $\lambda=\lambda_{0} \lambda_{1} \cdots \lambda_{i}$. If $\lambda_{i}=1$, then clearly $T(\lambda) \supseteqq L_{i-1}$ which is not the case. Hence $\lambda_{i} \neq 1$ and $B_{i} \neq 1$. The completes the forward half of the proof.

Conversely let $G$ have the structure described above. Since $A$ is abelian and $G / A$ is cyclic we know that there is no ramification. Let $\chi$ be an irreducible character of $G$ with $\lambda=\lambda_{0} \lambda_{1} \cdots \lambda_{k}$ a constituent of $\chi \mid A$. Then $\operatorname{deg} \chi=[G: T(\lambda)]$. If $\lambda=1$, then $\operatorname{deg} \chi=1=d_{0}$. If $\lambda \neq 1$ choose $j$ maximal with $\lambda_{j} \neq 1$. Clearly $T(\lambda)=L_{j}$ and $\operatorname{deg} \chi=d_{j}$. This completes the proof.

We now seek sufficient conditions to guarantee that $G / A$ is cyclic.

THEOREM 2.11. Each of the following will guarantee that $G / A$ is cyclic.

(i) $d_{k} \nmid d_{k-1}^{2}$

(ii) For all $i<j, q_{j} \nmid q_{i}$.

(iii) For all $i, q_{i+1}>q_{i}$.

(iv) There exists a prime $p \in \pi$ such that $\left|q_{i+1}\right|_{p}>\left|q_{i}\right|_{p}$ for all $i$.

Proof. We consider (i) first. This is a simple corollary of Theorem 2.6. If $G / A$ is not cyclic, then there exists $b<a<k$ with $\left(d_{k} / d_{a}\right) \mid\left(d_{a} / d_{b}\right)$. 
Thus $d_{k} \mid d_{a}^{2}$ and since $d_{a} \mid d_{k-1}$ this yields $d_{k} \mid d_{k-1}^{2}$, a contradiction.

Now assume $G$ satisfies condition (ii). We prove the result by induction on $|G|$. If $k=1$, the result follows by (i) above so we assume $k>1$. Let $\chi$ be a character of $G$ of degree $d_{1}$. By Lemma 2.8 (iii), $G / \operatorname{ker} \chi$ has characters of degrees 1 and $d_{1}$ only. Choose $K \triangle G, K \supseteqq \operatorname{ker} \chi$ with $G / K$ a Case $Q$ quotient. Using the notation of Lemma 2.7, it is clear that $[G: Q]=d_{1}=q_{1}$. Since $q_{j} \nmid d_{1}$ for all $j>1$, it follows by Lemma 2.7 that the distinct degrees of the characters of $Q$ are $d_{1} / d_{1}, d_{2} / d_{1}, \cdots, d_{k} / d_{1}$. Hence $Q$ has degree quotients $q_{2}, q_{3}, \cdots, q_{k}$ and we can apply induction to $Q$. Thus $Q / A$ is cyclic.

Theorem 2.10 applies to $Q$ and thus $A$ contains a characteristic subgroup $B$ on which $Q / A$ acts fixed point free. Then $B \triangle G$ and also $B \neq\langle 1\rangle$. Let $\lambda$ be a nonprincipal linear character of $B$ viewed as one of $A$. Then $\tilde{\lambda}$ (induction to $Q$ ) is an irreducible nonlinear character of $Q$ since $k>1$. By Lemma 2.7, $\tilde{\lambda}^{*}=\lambda^{*}$ is irreducible. This shows that $G / A$ acts fixed point free on $\hat{B}$ and hence $G / A$ is cyclic.

Parts (iii) and (iv) follow immediately from (ii).

3. Groups with a.c. $m$. In this section we study nonabelian groups $G$ having the property that every nonlinear irreducible character has degree $m$ for some fixed integer $m$. We say these groups have a.c. $m$ (all characters $m$ ). As an immediate consequence of Theorems 2.2 and 2.10 we have the following.

Theorem 3.1. Let $G$ have a.c. $m$ with $\pi=\pi(m)$. Suppose that either $|\pi|>1$ or $\pi=\{p\}$ and an $\mathfrak{S}_{p}$ subgroup of $G i$ is abelian. Then $G$ has the following structure.

(i) $G$ has a normal abelian $\mathfrak{S}_{\pi^{\prime}}$ subgroup $H \neq\langle 1\rangle$ with $G / H \cong$ $\mathfrak{S}_{\pi}(G)$ abelian.

(ii) $A=\mathfrak{C}(H)$ is a normal abelian subgroup of $G$ with $G / A$ cyclic of order $m$.

(iii) $A=3(G) \times B$ where $B \triangle G, B \neq\langle 1\rangle$ and $G / A$ acts fixed point free on $B$.

Conversely any group $G$ having this structure has a.c. $m$.

Therefore we need only consider the case in which $m=p^{e}$ for some prime $p$ with $\mathfrak{S}_{p}(G)$ nonabelian. Actually the $e=1$ case has already been studied in [2]. However there is little additional work involved in handling it so we will consider it again here. As we will see, the structure of those groups with $e>1$ is much more restrictive than the structure with $e=1$. We start with several lemmas.

Lemma 3.2. Let $G$ have a.c. $m$ with $m=p^{e}$ and $\mathfrak{S}_{p}(G)$ nonabelian. Then we have the following. 
(i) G has a normal abelian $\mathfrak{S}_{p^{\prime}}$ subgroup $H$.

(ii) $G$ has a Case $P$ quotient $E$.

(iii) If $E$ is any Case $P$ quotient of $G$, then $E$ is a p-group with $E / 3(E)$ elementary abelian of order $m^{2}$. Also any abelian subgroup $B$ of $E$ satisfies $[E: B] \geqq m$.

Proof. Since $G$ has r.x. $e$, Proposition 3.4 of [2] yields (i). Now $G / H \cong \mathfrak{S}_{p}(G)$ is nonabelian. Thus we can choose $K \triangle G, K \supseteqq H$ and maximal with $G / K$ nonabelian. Clearly $G / K$ is a Case $P$ quotient and (ii) follows. Now let $E$ be any Case $P$ quotient of $G$. Then $E$ has a.c. $m$ and hence $E$ is a $p$-group and $E / 3(E)$ is abelian of order $m^{2}$ by Proposition 2.2 of [2]. Since $E$ has an irreducible character of degree $m$, it follows that $E$ has no abelian subgroup of index less than $m$. We need only show that $E / 3(E)$ is elementary abelian. Given $x, y \in E$. Since $E$ has class 2 and $E^{\prime}$ has period $p$ we have $\left(x^{p}, y\right)=(x, y)^{p}=1$. Thus $x^{p} \in Z(E)$ and $E / 3(E)$ has period $p$.

Lemмa 3.3. Let $G$ have a.c. $m$. Then we have the following.

(i) Let $\mathscr{P}$ be a permutation representation of $G$ with deg $\mathscr{P} \leqq m$. Then $G^{\prime} \cong \operatorname{ker} \mathscr{P}$.

(ii) If $\left|G^{\prime}\right| \leqq m$, then $G^{\prime} \leqq 3(G)$.

(iii) Let $L$ be a subgroup of $G$ with $[G: L] \leqq m$. Then $G^{\prime} \leqq L$ and hence $L \triangle G$. Moreover if $[G: K]=m$ and $K<L \leqq G$, then $(K, L)=L^{\prime}=G^{\prime}$.

Proof. Let $\theta$ be the character corresponding to $\mathscr{P}$. Then $\operatorname{deg} \theta \leqq m$. We have $\theta=\sum a_{i} \chi_{i}$ where each $\chi_{i}$ is an irreducible character of $G$. Now if $\chi_{1}=1$, then $a_{1} \geqq 1$ and hence for all $i$, deg $\chi_{i}<m$. Since $G$ has a.c. $m, \operatorname{deg} \chi_{i}=1$ and $G^{\prime} \leqq \operatorname{ker} \chi_{i}$ and hence $G^{\prime} \subseteq \operatorname{ker} \mathscr{P}$. This yields (i).

Suppose $\left|G^{\prime}\right| \leqq m$. Let $x \in G$. Clearly $|C l x| \leqq\left|G^{\prime}\right| \leqq m$, where $C l x$ denotes the class of $x$. Now $G$ permutes the elements of $C l x$ by conjugation and this representation has degree $\leqq m$. Hence by (i) $G^{\prime}$ is in the kernel of the representation and thus $G^{\prime}$ centralizes $x$. Since $x$ was arbitrary, $G^{\prime} \leqq 3(G)$ and (ii) follows.

Now let $L \leqq G$ with $[G: L] \leqq m$. We see that $G$ permutes the right cosets of $L$ by right multiplication and this representation has degree $\leqq m$. Thus by (i), $G^{\prime}$ is in the kernel and hence $G^{\prime} \leqq L$. Now let $[G: K]=m$ and $L>K$. Since both $K$ and $L$ are normal in $G$ so is $H=(K, L)$. If $H<G^{\prime}$, then $G / H$ is nonabelian and thus has a.c. $m$. Since $K^{\prime} \subseteq H, K / H$ is abelian and is centralized by a properly larger subgroup. Thus $G / H$ has an abelian subgroup of index $<[G: K]=m$. This contradicts the existence of an irreducible character of $G / H$ of degree $m$. Hence $H=G^{\prime}$. Since $G^{\prime} \supseteqq L^{\prime} \supseteqq(K, L)$, the result follows. 
Lemma 3.4. Let $G$ have a.c. $m$ where $m=p^{e}$ and $\Im_{p}(G)$ is nonabelian. Let $A \supseteqq H=\mathfrak{S}_{p^{\prime}}(G)$ be a normal self-centralizing subgroup of $G$. If $G$ has a faithful irreducible character $\chi$ then $G / A$ is elementary abelian of order $m$.

Proof. Since $A \supseteqq H, G / A$ is a $p$-group and hence there exists subgroup $L$ and linear character $\lambda$ of $L$ with $G \supseteqq L \supseteqq A$ and $\chi=\lambda^{*}$. Since $\operatorname{deg} \chi=m,[G: L]=m$ and hence by Lemma 3.3 (iii), $L \triangle G$. Thus $\chi \mid L$ has only linear constituents. Since $\chi$ is faithful $L$ is abelian and since $A$ is self-centralizing $L=A$. Thus $G / A$ has order $m$.

By Lemma 3.2(ii), $G$ has a Case $P$ quotient $E=G / K$. Let $Z / K=$ $3(E)$. Then $(A Z) / K$ is an abelian subgroup of $E$ so $[G: A Z] \geqq m$ by Lemma 3.2 (iii). Since $[G: A]=m$, we have $A \supseteqq Z$ and hence $G / A$ is elementary abelian.

We now reduce the study of these groups to a study of $p$-groups.

Theorem 3.5. Let $G$ have a.c. $p^{e}$ with $P=\mathfrak{S}_{p}(G)$ nonabelian. Let $H$ be the normal abelian $\mathfrak{S}_{p^{\prime}}$ subgroup.

(i) If $e>1$, then $H$ is central and hence $G=H \times P$.

(ii) If $e=1$, then either $H$ is central or $G$ has a normal abelian subgroup of index $p$.

Proof. We start with the case $e>1$. Suppose first that $G$ has a faithful irreducible character. By the preceeding lemma, $G$ has a normal abelian subgroup $A$ with $G / A$ elementary abelian of order $p^{e}$. Then $A=B \times H$ where $B=\mathfrak{S}_{p}(A) \triangle G$. We consider $G / B$ and show it is abelian. If not, then $G / B$ has a.c. $p^{e}$. Now $\mathfrak{S}_{p}(G / B) \cong G / A$ is abelian and thus Theorem 3.1 applies. Hence since $A / B=\mathfrak{S}_{p^{\prime}}(G / B)$ we see that $(G / B) / \mathbb{C}(A / B)$ is cyclic of order $p^{e}$. This implies that $\mathfrak{S}(A / B)=$ $A / B$ and therefore that $G / A$ is cyclic of order $p^{e}$. Since $G / A$ is elementary abelian this is a contradiction for $e>1$. Thus $G / B$ is abelian. Since $H \triangle G$ this yields $(G, H) \subseteq B \cap H=\langle 1\rangle$ and $H$ is central.

Now let $G$ be arbitrary with $e>1$. We show that $H$ is central. If not choose $x \in P^{\prime}, y \in(G, H)$ with $x, y \neq 1$. By Proposition 4.6 of [2] there exists an irreducible character $\chi$ of $G$ with $x, y \notin \operatorname{ker} \chi$. Hence $G / \operatorname{ker} \chi$ has a. c. $p^{e}$, a nonabelian Sylow $p$-subgroup and a noncentral $\mathfrak{S}_{p^{\prime}}$ subgroup. Since $G / \operatorname{ker} \chi$ has a faithful character this contradicts the above and (i) is proved.

Now let $e=1$ and suppose that $H$ is not central. Since $p>1=e$, $G$ is imprimitive by Theorem 1.1. Thus there exists $A \triangle G$ with $[G: A]=$ $p$ such that $e(A)=e(G)-1=0$. Hence $A$ is abelian and (ii) follows.

It is easy to construct examples to show that $H$ need not be central 
in $G$ if $e=1$. For example, let $Q$ be an abelian $q$-group $(q \neq p)$ which has an automorphism of order $p$. Let $G=Q \times{ }_{\sigma} P$ where $P$ has order $p^{3}$ and $P$ acts on $Q$ in such a way that $P_{0}$ a subgroup of index $p$ centralizes $Q$ and $P / P_{0}$ corresponds to the automorphism of order $p$. Since $A=Q P_{0}$ is an abelian subgroup of $G$ of index $p, G$ has a.c. $p$. Finally $Q=\mathfrak{S}_{p^{\prime}}(G)$ is not central and $P=\mathfrak{S}_{p}(G)$ can be chosen to be nonabelian.

In the remainder of this section and in the next two sections we will consider only $p$-groups.

Lemma 3.6. Let $G$ have a.c. $m$. Then we have the following.

(i) $\Phi(G)$, the Frattini subgroup of $G$, is abelian.

(ii) If $G$ has two distinct abelian subgroups $A$ and $B$ of index $m$, then $\left|G^{\prime}\right| \leqq m$ and hence $G$ has class 2 . Moreover if $\left|G^{\prime}\right|=m$, then $3(G)=A \cap B$ and $[G: 3(G)]=m^{2}$.

(iii) If $G^{\prime}$ is not central, then $\mathfrak{S}\left(G^{\prime}\right)$ is abelian.

Proof. We consider (ii) first. Choose $x \in B-A$ with $x^{p} \in A$ and set $L=\langle A, x\rangle$. Then $[G: L]=p^{e-1}$ where $m=p^{e}$. Thus by Lemma 3.3 (iii), $L^{\prime}=G^{\prime}$. Clearly $L^{\prime}=(A, x)$ and $\left|G^{\prime}\right|=\left|L^{\prime}\right|=\left[A: \widetilde{夭}_{A}(x)\right]$. Since $\mathfrak{F}_{A}(x) \supseteqq A \cap B$ and $[A: A \cap B] \leqq m$, we have $\left|G^{\prime}\right| \leqq m$. By Lemma 3.3(ii), $G$ has class 2. If $\left|G^{\prime}\right|=m$, then $[A: A \cap B]=m$ and so $[A B: B]=m$. Thus $G=A B$ and since $A$ and $B$ are abelian $B(G) \supseteq A \cap B$. On the other hand $A$ and $B$ must be maximal abelian subgroups so $A, B \supseteqq Z(G)$. Thus $3(G)=A \cap B$ and (ii) follows.

If $\Phi(G)$ is not abelian, then there exists an irreducible character $\chi$ of $G$ with $\Phi^{\prime} \nsubseteq \operatorname{ker} \chi$. Hence $\Phi(G / \operatorname{ker} \chi)$ is nonabelian. Now $\bar{G}=G / \operatorname{ker} \chi$ has a normal abelian subgroup $A$ with $\bar{G} / \bar{A}$ elementary abelian by Lemma 3.4. Hence $\Phi(\bar{G}) \subseteq \bar{A}$, a contradiction and (i) follows.

Now assume $G^{\prime}$ is not central. If $\mathbb{E}\left(G^{\prime}\right)$ is not abelian we can choose $x, y \in \mathbb{C}\left(G^{\prime}\right)$ with $(x, y) \neq 1$. Choose $z \in\left(G, G^{\prime}\right)$ with $z \neq 1$. Then there exists an irreducible character $\chi$ of $G$ with $(x, y), z \notin \operatorname{ker} \chi$. Therefore it suffices to assume that $G$ has a faithful irreducible character. Since $G^{\prime}$ is abelian, we can extend normal abelian subgroups $\left\langle x, G^{\prime}\right\rangle$ and $\left\langle y, G^{\prime}\right\rangle$ to normal self-centralizing subgroups $A$ and $B$. By Lemma 3.4, $[G: A]=[G: B]=m$. Since $x \in A, y \in B$ and $(x, y) \neq 1$, we see that $A \neq B$. By (ii) above $G$ has class 2 , a contradiction. This completes the proof of the lemma.

THEOREM 3.7. Let $G$ be a p-group with a.c.m. Then either $G$ has a normal abelian subgroup $A$ with $G / A$ elementary abelian of order $m$ or $G$ has class at most 3 .

Proof. By induction on $|G|$. If $3(G)$ is cyclic, then $G$ has a 
faithful irreducible character and the result follows by Lemma 3.4. Hence we can assume that $3(G)$ is not cyclic and thus $3(G)$ has at least three distinct subgroups $J_{1}, J_{2}, J_{3}$ of order $p$. We can clearly assume that $G$ has class $>3$. Since the subgroups $J_{i}$ are disjoint it follows that at most one quotient $G / J_{i}$ has class $\leqq 3$. Hence say $G / J_{1}$ and $G / J_{2}$ have class $>3$. By induction, for $i=1,2, G / J_{i}$ has a normal abelian subgroup $A_{i} / J_{i}$ with $G / A_{i}$ elementary abelian of order $m$. Set $U=J_{1} J_{2}$ so that $U \subseteq 3(G)$. If $A_{1} \neq A_{2}$, then $A_{1} / U$ and $A_{2} / U$ are two distinct abelian subgroups of $G / U$ of index $m$. By Lemma 3.6 (ii), $G / U$ has class $\leqq 2$ and thus $G$ has class $\leqq 3$, a contradiction. Therefore $A_{1}=A_{2}=A$. Since $A_{i} / J_{i}$ is abelian, $A^{\prime} \leqq J_{1} \cap J_{7}=\langle 1\rangle$ and $A$ is abelian. This completes the proof.

Let $\gamma^{i} G$ denote the $i$ th term of the lower central series of $G$. Thus $\gamma^{0} G=G, \gamma^{i+1} G=\left(\gamma^{i} G, G\right)$ and the class of $G$ is the minimal $c$ with $\gamma^{c} G=\langle 1\rangle$.

LEMMA 3.8. (i) Let $G$ be an arbitrary p-group having a normal abelian subgroup $A$ with $G / A$ abelian of order $m$. Suppose for all subgroups $H$ with $G \supseteqq H>A$ and $[H: A]=p$ we have $H^{\prime}=G^{\prime} \neq\langle 1\rangle$. Then $G$ has a.c.m.

(ii) Let $G$ have a.c. $m$. and a normal abelian subgroup $A$ with $G / A$ abelian of order $m$. If $G^{\prime}$ is not central, then $K=G^{\prime} \times_{o}(G / A)$ has a.c.m. Moreover let $x \in G / A$ have order $p$. Then for all $i>0$, $\gamma^{i} G=A^{(1-x)^{i}}$.

Proof. We consider (i). Let $\chi$ be an irreducible character of $G$. Since $[G: A]=m$ we have $\operatorname{deg} \chi \leqq m$. We assume $\operatorname{deg} \chi<m$. Since $A$ is normal and abelian, $\chi$ is induced from a linear character of some subgroup $L \supseteq A$. Clearly $L>A$ and we can choose $H$ with $L \supseteq H>A$ and $[H: A]=p$. Since $G / A$ is abelian, $L \triangle G$. Thus ker $\chi \supseteqq L^{\prime} \supseteqq H^{\prime}$ and since $H^{\prime}=G^{\prime}$, $\operatorname{ker} \chi \supseteqq G^{\prime}$. Thus $\operatorname{deg} \chi=1$. Since $\left|G^{\prime}\right| \neq 1, G$ has a.c. $m$.

Now let $G$ have a.c. $m$ and a normal abelian subgroup $A$ of index $m$. Set $K=G^{\prime} \times_{o}(G / A)$. Then $K$ has a normal abelian subgroup $G^{\prime}$ with $K / G^{\prime} \cong G / A$ abelian of order $m$. Let $x$ have order $p$ in $G / A$ and set $H=\langle A, y\rangle$ where $y$ is an inverse image of $x$ in $G$. Then $H \triangle G$ and $\left(x, G^{\prime}\right)=\left(H, G^{\prime}\right) \triangle G$. Now $\bar{G}=G /\left(x, G^{\prime}\right)$ is nonabelian since $\left(x, G^{\prime}\right)<G^{\prime}$ and thus $\bar{G}$ has a.c. $m$. Clearly $\subseteq\left(\bar{G}^{\prime}\right) \supseteqq H /\left(x, G^{\prime}\right)$ so $\left[\bar{G}: \mathfrak{S}\left(\bar{G}^{\prime}\right)\right]<m$. Now $\bar{G}$ cannot have an abelian subgroup of index $<m$ and hence by Lemma 3.6 (iii), $\bar{G}^{\prime} \subseteq 3(\bar{G})$. Therefore $\left(x, G^{\prime}\right)=\left(G, G^{\prime}\right)=$ $\left(G / A, G^{\prime}\right)$. Thus we see that $K$ satisfies the hypothesis of (i) above, since $\left(G, G^{\prime}\right) \neq\langle 1\rangle$ by assumption. Thus $K$ has a.c. $m$.

Let $K$ be as above. We know that for $i \geqq 1$ 


\section{and}

$$
\begin{aligned}
\gamma^{i} G & =(A, G / A, G / A, \cdots, G / A) \\
\gamma^{i} K & =\left(G^{\prime}, G / A, G / A, \cdots, G / A\right)
\end{aligned}
$$

where $G / A$ occurs $i$ times in each of the above. This follows using Lemma 3.3 (iii) for $i=1$. Since $G^{\prime}=(A, G / A)$ we have for $i \geqq 2$, $\gamma^{i} G=\gamma^{i-1} K$.

Let $x \in G / A$ have order $p$. We show that for $i \geqq 1, \gamma^{i} G=A^{(1-x)^{i}}$ by induction on $i$. If $i=1$, the result follows from Lemma 3.3 (iii). Let $i \geqq 2$ so that $\gamma^{i} G=\gamma^{i-1} K$. By induction, since $i-1 \geqq 1$, we have $\gamma^{i-1} K=\left(G^{\prime}\right)^{(1-x)^{i-1}}$. Since $G^{\prime}=A^{(1-x)}$ the result follows.

EXAMPLE 3.9. Let $D$ be an additive elementary abelian group of order $m=p^{e}$ and let $A_{1}, A_{2}, \cdots, A_{p}$ be $p$ distinct groups isomorphic to $D$. Say $\alpha_{i}: D \rightarrow A_{i}$ is an isomorphism.

Let $F$ be a field of endomorphisms of $D$ with $|F|=m$. In fact $F$ corresponds to the regular representation of $G F\left(p^{e}\right)$ on its additive group. For $\sigma \in F$ define $\sigma_{i}: A_{i} \rightarrow A_{i+1}$ by $\sigma_{i}\left(a_{i}\right)=\alpha_{i+1} \sigma \alpha_{i}^{-1}\left(\alpha_{i}\right)$ for $i=$ $1,2, \cdots, p-1$ and $\sigma_{p}: A_{p} \rightarrow\langle 0\rangle$. Let $A=A_{1}+A_{2}+\cdots+A_{p}$ and define $\bar{\sigma}$ on $A$ by $\bar{\sigma}=\sigma_{1}+\sigma_{2}+\cdots+\sigma_{p}$. Clearly $\bar{\sigma}^{p}=0$.

Let $\sigma, \tau \in F$. We show that $\bar{\sigma} \bar{\tau}=\bar{\tau} \bar{\sigma}$. Let $a_{i} \in A_{i}$. If $i=p-1$ or $p$ then $\bar{\sigma} \bar{\tau}\left(a_{i}\right)=0=\bar{\tau} \bar{\sigma}\left(a_{i}\right)$. Now let $i<p-1$. Then

$$
\bar{\sigma} \bar{\tau}\left(a_{i}\right)=\sigma_{i+1} \tau_{i}\left(a_{i}\right)=\alpha_{i+2} \sigma \alpha_{i+2}^{-1} \alpha_{i+1} \tau \alpha_{i}^{-1}\left(a_{i}\right)=\alpha_{i+2} \sigma \tau \alpha_{i}^{-1}\left(a_{i}\right) .
$$

Since $\sigma \tau=\tau \sigma$ we have clearly $\bar{\sigma} \bar{\tau}=\bar{\tau} \bar{\sigma}$.

Now for $\sigma \in F$ set $x_{\sigma}=1+\bar{\sigma}$. Since $\bar{\sigma}^{p}=0$ and $A$ is elementary abelian we have $x_{\sigma}^{p}=1$. Also for $\sigma, \tau \in F$ we have $x_{\sigma} x_{\tau}=x_{\tau} x_{\sigma}$.

Let $\sigma_{1}, \sigma_{2}, \cdots, \sigma_{e}$ be a basis of $F$ over $G F(p)$ and set $x_{i}=x_{\sigma_{i}}$ for convenience. Let $B$ be the elementary abelian group of automorphisms of $A$ generated by the $x_{i}$. Clearly $|B| \leqq p^{e}$. Set $S=\left\{x_{\sigma}\right\}$. Since $x_{\sigma} x_{\tau}=1+\bar{\sigma}+\bar{\tau}+\bar{\sigma} \bar{\tau}$ it follows that when restricted to

$$
\bar{A}_{i}=\left(A_{i}+A_{i+1}+\cdots+A_{p}\right) /\left(A_{i+2}+\cdots+A_{p}\right)
$$

$S$ is a group of order $p^{e}$. Here $i=1, \cdots, p-1$ and if $i=p-1$ then the denominator of the quotient is the group $\langle 0\rangle$. Clearly $B$ and $S$ restricted to this quotient are isomorphic and hence $|B|=p^{e}$.

Now let $x \in B$ with $x \neq 1$. Then there exists $\sigma \in F, \sigma \neq 0$ such that $x$ and $x_{\sigma}$ act the same way on $\bar{A}_{i}$ above for all $i$. Since $\sigma$ is an onto map we see that

$$
\begin{gathered}
{\left[(1-x)\left(A_{i}+A_{i+1}+\cdots+A_{p}\right)\right]\left(A_{i+2}+\cdots+A_{p}\right)} \\
=\left(A_{i+1}+\cdots+A_{p}\right)
\end{gathered}
$$

for $i=1,2, \cdots, p-1$. This clearly yields

$$
(1-x) A=\left(A_{2}+A_{3}+\cdots+A_{p}\right) .
$$


Thus by Lemma 3.8(i), $G=A \times{ }_{\rho} B$ has a.c.m. Moreover as is easily seen, $G$ has class $p$.

The following result exhibits another difference between the $e=1$ and $e>1$ cases.

Theorem 3.10. Let $G$ have a.c.p with $e>1$. Then $G$ has class at most $p$ and $G^{\prime}$ is elementary abelian.

Proof. By induction on $|G|$. If $3(G)$ is not cyclic choose $J_{1}, J_{2}$ subgroups of $3(G)$ with $J_{1} \cap J_{2}=\langle 1\rangle$. By induction $G / J_{i}$ has class $\leqq p$ so $\gamma^{p} G \leqq J_{1} \cap J_{2}=\langle 1\rangle$. Also $\left(G / J_{i}\right)^{\prime}$ has period $p$ so clearly $G^{\prime}$ is elementary abelian.

Now assume $3(G)$ is cyclic. By Lemma 3.4, $G$ has a normal abelian subgroup $A$ with $G / A$ elementary abelian of order $p^{e}$. Let $H=G / A$ so that $I(H)$, the group ring of $H$ over the rational integers $I$, acts on $A$. If $S$ is a subset of $H$ we let $\widetilde{S}$ denote the sum of the elements of $S$ in $I(H)$. Let $K$ be a nonidentity subgroup of $H$. Choose $x \in K$ with $x \neq 1$. By Lemma 3.8 (ii), $G^{\prime}=A^{(1-x)}$. Hence

$$
\left(G^{\prime}\right)^{\widetilde{K}}=A^{(1-x) \widetilde{K}}=\langle 1\rangle
$$

since $(1-x) \widetilde{K}=0$ in $I(H)$. Thus $\widetilde{K}$ annihilates $G^{\prime}$.

Since $e \geqq 2$ we can choose $K$ to be a subgroup of $H$ of order $p^{2}$. Let $K_{0}, K_{1}, \cdots, K_{p}$ be the subgroups of $K$ of order $p$. Note that $K$ is elementary abelian. Now in $I(H)$

$$
p=\left(\sum_{0}^{p} \widetilde{K}_{i}\right)-\widetilde{K}
$$

and hence $p$ annihilates $G^{\prime}$. Thus $G^{\prime}$ has period $p$.

Now let $J$ be a subgroup of $H$ of order $p$ with $J=\langle x\rangle$. Then as is well known

$$
\widetilde{J}=1+x+\cdots+x^{p-1} \equiv(1-x)^{p-1} \bmod p I(H) .
$$

By Lemma 3.8 (ii),

$$
\gamma^{p} G=A^{(1-x)^{p}}=A^{(1-x)(1-x)^{p-1}}=\left(G^{\prime}\right)^{(1-x)^{p-1}} .
$$

Since $G^{\prime}$ has period $p$ we can take $(1-x)^{p-1}$ modulo $p I(H)$ in the above. Therefore $\gamma^{p} G=\left(G^{\prime}\right)^{\widetilde{T}}=\langle 1\rangle$ and $G$ has class $\leqq p$. This completes the proof.

ExAmPLe 3.11. If $e=1$, the above result is false. For example, let $A=\left\langle\alpha_{1}\right\rangle \times\left\langle\alpha_{2}\right\rangle \times \cdots \times\left\langle\alpha_{p}\right\rangle$ where each $\alpha_{i}$ has order $p^{a}$. Let $J=\langle x\rangle$ be cyclic of order $p$ and let $J$ act on $A$ by $\alpha_{i}^{x}=\alpha_{i+1}$ for $i=1,2, \cdots, p-1$ and $\alpha_{p}^{X}=\alpha_{1}$. If $G=A \times_{\sigma} J$, then $G$ has a.c.p. 
Now $\alpha_{2} \alpha_{1}^{-1} \in G^{\prime}$ and hence if $a>1$ we see that $G^{\prime}$ is not elementary abelian. Moreover as we see below $G$ has class $a(p-1)+1$. First in $I(J)$

$$
(1-x)^{p-1}-\widetilde{J} \equiv 0 \quad \bmod p I(J)
$$

and hence

$$
\left[(1-x)^{p-1}-\widetilde{J}\right]^{a} \equiv 0 \quad \bmod p^{a} I(J) .
$$

Since $(1-x) \widetilde{J}=\widetilde{J}(1-x)=0$ and $(\widetilde{J})^{2}=p \widetilde{J}$, the above yields

$$
(1-x)^{a(p-1)} \equiv(-p)^{a-1} \widetilde{J} \quad \bmod p^{a} I(J) .
$$

Now $A$ has period $p^{a}$ and hence $(1-x)^{a(p-1)}$ and $(-p)^{a-1} \widetilde{J}$ act the same way on $A$. Since $\widetilde{J}(1-x)=0$ we see from the nature of the action of $x$ on $A$ that

and

$$
\begin{gathered}
\gamma^{a(p-1)} G=A^{(1-x)^{a(p-1)}}=A^{(-p)^{a-1} \widetilde{J}} \neq\langle 1\rangle \\
\gamma^{a(p-1)+1} G=A^{(-p)^{a-1} \widetilde{J}(1-x)}=\langle 1\rangle .
\end{gathered}
$$

Hence $G$ has class $a(p-1)+1$ and this can be arbitrarily large.

4. Special class 3 groups. Let $G$ be a $p$-group with a.c. $p^{e}$. We say that $G$ is special if it does not have a normal abelian subgroup of index $p^{e}$. By Theorem 3.7 if $G$ is special, then $G$ has class 2 or 3 . As is expected the structure of the special class 3 groups is quite restrictive. We study these latter groups in this section.

THeOREM 4.1. Let $G$ be a special class 3 group with a.c.m. Then we have the following.

(i) $\left[G^{\prime}: \gamma^{2} G\right]=m$ and $\gamma^{2} G=G^{\prime} \cap 3(G)$.

(ii) $\left[G: \mathbb{E}\left(G^{\prime}\right)\right]=m^{2}$ and $\mathbb{E}\left(G^{\prime}\right)$ is a normal self-centralizing subgroup.

(iii) $[G: 3(G)]=m^{3}$.

(iv) If $H=G / 3(G)$, then $H^{\prime}=3(H)$ is elementary abelian of order $m$ and $H$ has two normal abelian subgroups of index $m$ whose intersection is equal to $H^{\prime}$.

We start with a lemma.

LEMMA 4.2. Let $G$ have a.c. $m$ and class 3 .

(i) If $\gamma^{2} G$ is cyclic or if $\left[G^{\prime}: \gamma^{2} G\right]>m$, then $G$ has an abelian subgroup $A$ of index $m$.

(ii) $\left[G^{\prime}: G^{\prime} \cap 3(G)\right] \geqq m$ and $[G: 3(G)] \geqq m^{3}$.

Proof. By induction on $|G|$. Suppose that $\gamma^{2} G$ is cyclic. Then 
there exists an irreducible character $\chi$ of $G$ with $\gamma^{2} G \cap \operatorname{ker} \chi=\langle 1\rangle$. By Lemma 3.4, $G /$ ker $\chi$ has an abelian subgroup $A / \operatorname{ker} \chi$ of index $m$ with $A \supseteqq G^{\prime}$. Then $\left(A, G^{\prime}\right) \subseteq \gamma^{2} G \cap \operatorname{ker} \chi=\langle 1\rangle$ so $A \cong \mathbb{E}\left(G^{\prime}\right)$. Since $G$ has class larger than $2, \mathfrak{S}\left(G^{\prime}\right)$ is abelian by Lemma 3.6 (iii) and hence $A$ is abelian.

Now suppose $\left[G^{\prime}: \gamma^{2} G\right]>m$. If $\gamma^{2} G$ is cyclic, then the result follows by the above. Thus we can assume that $\gamma^{2} G$ contains distinct subgroups $J_{1}$ and $J_{2}$ of order $p$. Since $\gamma^{2} G \subseteq 3(G), J_{1}$ and $J_{2}$ are normal in $G$. By induction $G / J_{i}$ has an abelian subgroup $A_{i} / J_{i}$ of index $m$. Set $U=J_{1} J_{2} \subseteq \gamma^{2} G$. If $A_{1} \neq A_{2}$, then $A_{1} / U$ and $A_{2} / U$ are two distinct abelian subgroups of $G / U$ of index $m$. Hence $\left|(G / U)^{\prime}\right| \leqq m$ by Lemma 3.6 (ii). Since $U \subseteq \gamma^{2} G$ this yields $\left[G^{\prime}: \gamma^{2} G\right] \leqq m$, a contradiction. Thus $A_{1}=A_{2}=A$ and hence $A^{\prime} \subseteq J_{1} \cap J_{2}=\langle 1\rangle$. Therefore $A$ is abelian and (i) follows.

We consider (ii). The result is obvious if $m=p$ and hence we assume $m=p^{e}$ with $e>1$. By Theorem $3.10 G^{\prime}$ is elementary abelian. If $G^{\prime} \cap 3(G)$ is not cyclic, there exists subgroup $J$ of $G^{\prime} \cap 3(G)$ with $J \nsupseteq \gamma^{2} G$. Hence $\bar{G}=G / J$ has class 3 . By induction $\left[\bar{G}^{\prime}: \bar{G}^{\prime} \cap 3(\bar{G})\right] \geqq m$. Now $\bar{G}^{\prime}=G^{\prime} / J$ and $\bar{G}^{\prime} \cap 3(\bar{G}) \supseteqq\left(G^{\prime} \cap 3(G)\right) / J$. Thus the result follows in this case. Now let $G^{\prime} \cap 3(G)$ be cyclic. Since $G^{\prime}$ is elementary abelian $\left|G^{\prime} \cap 3(G)\right|=p$. Now $G$ has class $>2$ and thus by Lemma 3.3 (ii), $\left|G^{\prime}\right| \geqq p m$. Hence $\left[G^{\prime}: G^{\prime} \cap 3(G)\right] \geqq m$.

Let $W / \gamma^{2} G$ be the center of $G / \gamma^{2} G$. Since $G / \gamma^{2} G$ has a.c. $m$ we see that $[G: W] \geqq m^{2}$. Clearly $3(G) \subseteq W$ and $G^{\prime} \leqq W$. Hence

$$
[W: 3(G)] \geqq\left[W \cap G^{\prime}: 3(G) \cap G^{\prime}\right]=\left[G^{\prime}: 3(G) \cap G^{\prime}\right] \geqq m .
$$

Therefore $[G: 3(G)]=[G: W][W: 3(G)] \geqq m^{3}$ and the lemma is proved.

Proof of Theorem 4.1. We assume throughout that $G$ is a special class 3 group with a.c. $m$. Since $\gamma^{2} G \subseteq G^{\prime} \cap 3(G)$ we have $\left[G^{\prime}: \gamma^{2} G\right] \geqq m$ by Lemma 4.2 (ii). Moreover since $G$ is special $\left[G^{\prime}: \gamma^{2} G\right] \leqq m$ by Lemma 4.2 (i). Hence $\left[G^{\prime}: \gamma^{2} G\right]=m$ and (i) follows.

Let $K_{1}, K_{2}, \cdots, K_{s}$ be all the proper subgroups of $\gamma^{2} G$ with $\gamma^{2} G / K_{i}$ cyclic. Clearly $\cap K_{i}=\langle 1\rangle$. By the preceeding lemma, $G / K_{i}$ has a normal abelian group $B_{i} / K_{i}$ of index $m$. By Lemma 3.3 (iii) $B_{i} \supseteqq G^{\prime}$. Since $G / K_{i}$ has class 3 , Lemma 3.6 (iii) yields $B_{i} / K_{i}=\mathbb{E}\left(G^{\prime} / K_{i}\right)$. Thus for all $i, B_{i} \supseteqq A=\mathbb{E}\left(G^{\prime}\right)$. Set $B=\bigcap B_{i}$ so that $B \supseteqq A$. Since $\left(B, G^{\prime}\right) \cong K_{i}$ we have $\left(B, G^{\prime}\right) \leqq \bigcap K_{i}=\langle 1\rangle$. Thus $B=A$.

Choose $L \triangle G$ with $G / L$ a Case $P$ quotient. Let $Z / L$ be the center of $G / L$ so that $[G: Z]=m^{2}$. Clearly $L \supseteqq \gamma^{2} G$. Since $B_{i} Z / L$ is an abelian subgroup of $G / L$ of index $\leqq m$ we must have $B_{i} \supseteqq Z$ by Lemma 3.2 (iii). Thus $B \supseteqq Z$ and $[G: A] \leqq m^{2}$. Now if $B=B_{1}$, then clearly $B$ is an abelian subgroup of $G$ of index $m$ and this does not occur. 
Thus say $B_{1} \neq B_{2}$. Then $G / \gamma^{2} G$ has two distinct abelian subgroups $B_{1} / \gamma^{2} G$ and $B_{2} / \gamma^{2} G$ of index $m$. Since $\left|G^{\prime} / \gamma^{2} G\right|=m$ we see that $\left[G: B_{1} \cap B_{2}\right]=m^{2}$ by Lemma 3.6 (ii). Hence $[G: A]=m^{2}$. This proves (ii) and the part of (iv) concerning the existence of two abelian subgroups of $H$ of index $m$.

We prove (iii) by induction on $|G|$. Say $\left|\gamma^{2} G\right|=p^{r}$. By the preceeding lemma $\gamma^{2} G$ is not cyclic and hence $r \geqq 2$. Let $J$ be a subgroup of $\gamma^{2} G$ of order $p$. Suppose that $G / J$ has an abelian subgroup $B / J$ of index $m$. Then $B$ is nonabelian so $B^{\prime}=J$ and $B$ has class 2. Clearly $B \supseteqq \mathbb{C}\left(G^{\prime}\right)$ and $\mathbb{C}\left(G^{\prime}\right)$ is a maximal normal abelian subgroup of $B$. Since $\left[B: \mathfrak{c}\left(G^{\prime}\right)\right]=m$, it follows that $B$ has a.c. $m$ and $[B: 3(B)]=m^{2}$ by Lemma 2.3 of [2]. Let $x \in B$ with $x \neq 1$. Then there exists an irreducible character $\chi$ of $B$ with $x, y \notin \operatorname{ker} \chi$ where $J=\langle y\rangle$. Hence $\chi$ is nonlinear and $\operatorname{deg} \chi=m$. This says that $B$ is character regular. Since $e(B)=e(G)=e$ where $m=p^{e}$, it follows by Proposition 1.3 (i) that $3(B) \subseteq 3(G)$. Since clearly $3(G) \subseteq \mathbb{}\left(G^{\prime}\right) \subseteq B$ we have $3(G)=$ $3(B)$ and thus $[G: 3(G)]=[G: B][B: 3(G)]=m^{3}$. Thus the result follows in this case. Note that if $r=2$ the $\gamma^{2}(G / J)$ is cyclic so the result follows here.

We assume that $r \geqq 3$ and that for all subgroups $J$ of $\gamma^{2} G$ of order $p$ the quotient $G / J$ is a special class 3 group. Since $\gamma^{2} G$ is not cyclic, let $J_{1}$ and $J_{2}$ be two such subgroups of order $p$ and set $U=J_{1} J_{2}$. Thus $|U|=p^{2}<p^{r}=\left|\gamma^{2} G\right|$ and $U<\gamma^{2} G$. By induction $G / J_{i}$ has center $Z_{i} / J_{i}$ of index $m^{3}$ If $Z_{1} \neq Z_{2}$ then we see that $\left(Z_{1} Z_{2}\right) / U$ is central in $G / U$ and has index $<m^{3}$. Since $U<\gamma^{2} G, G / U$ has class 3 and a.c. $m$ and this violates Lemma 4.2 (ii). Thus $Z_{1}=Z_{2}=Z$. Since $\left(Z_{i}, G\right) \leqq J_{i}$ it follows that $(Z, G) \subseteq J_{1} \cap J_{2}=\langle 1\rangle$ and hence $Z=3(G)$. This yields (iii).

Finally we know that $|H|=m^{3},[H: 3(H)] \geqq m^{2}, 3(H) \supseteq H^{\prime}$ and $\left|H^{\prime}\right| \geqq m$. The latter follows since $\left[G^{\prime}: G^{\prime} \cap 3(G)\right] \geqq m$. Hence we must have equality throughout. Now $H$ has a.c. $m$ with $m=p^{e}$. If $e>1$, then $H^{\prime}$ is elementary abelian by Theorem 3.10. If $e=1$, then $\left|H^{\prime}\right|=p$ and the result is clear here. Thus the theorem is proved.

We used simple facts about $G F\left(p^{e}\right)$ to obtain Example 3.9. In order to construct special class 3 groups we will need the following interesting fact about these fields. The authors would like to thank Walter Feit for his help with the proof of this result.

Proposition 4.3. Let $E$ be a finite field of characteristic $p>2$ and let $F$ be a subfield. Then there exists a basis of $E$ over $F$ with respect to which every matrix of the regular representation of $E$ over $F$ is symmetric. 
Proof. Let $w=\left\{w_{1}, w_{2}, \cdots, w_{n}\right\}$ be a basis of $E$ over $F$ and let $R_{w}$ be the matrix form of the regular representation with respect to this basis. Let $\theta \in E$ be a primitive element so that $E=F(\theta)$. Then the characteristic polynomial of $R_{w}(\theta)$ is irreducible over $F$. Note that all matrices below are over $F$.

By Theorem 1 of [8] there exists a matrix $S$ with $S^{-1} R_{w}(\theta) S=$ $R_{w}(\theta)^{\prime}$. Here ' denotes the transpose operation. As is well known the norm map from $E$ to $F$ is onto and hence there exists $\alpha \in E$ with $\operatorname{det} S=N_{E \mid F}(\alpha)=\operatorname{det} R_{w}(\alpha)$. If $T=R_{w}\left(\alpha^{-1}\right) S$, then $T^{-1} R_{w}(\theta) T=$ $R_{w}(\theta)^{\prime}$ since $R_{w}(\alpha)$ and $R_{w}(\theta)$ commute. Moreover $\operatorname{det} T=1$. By Theorem 2 of [8], $T$ is symmetric.

Now $T$ is symmetric and $\operatorname{det} T=1$, a square in $F$. Since $F$ is a finite field of characteristic $p>2$, there exists a matrix $U$ with $T=$ $U U^{\prime}$. Let $A=U^{-1} R_{w}(\theta) U$. Then

$$
\begin{aligned}
A^{\prime} & =U^{\prime} R_{w}(\theta)^{\prime}\left(U^{\prime}\right)^{-1}=U^{\prime} T^{-1} R_{w}(\theta) T\left(U^{\prime}\right)^{-1} \\
& =U^{-1} R_{w}(\theta) U=A .
\end{aligned}
$$

Hence if we let $U$ be a change of basis matrix, $U: w \rightarrow v$, then $A=$ $R_{v}(\theta)$ is symmetric. Since $E=F(\theta)$, the result follows.

THEOREM 4.4. Special class 3 groups with a.c.p $p^{e}$ exist for all $p>2$ and $e$. No such groups exist for $p=2$.

Proof. Let $p=2$. If $e>1$, then by Theorem 3.10 groups $G$ with a.c. $p^{e}$ have class $\leqq p=2$. Hence no special class 3 groups exist. If $e=1$ and $G$ is a special class 3 group, then $[G: 3(G)]=8$ by Theorem 4.1. Therefore $H=G / 3(G)$ is nonabelian of order 8. Such groups all have cyclic subgroups of order 4 . Thus if $A<G$ with $A / 3(G)$ cyclic of order 4, then $A$ is an abelian subgroup of $G$ of index 2 and hence $G$ is not special, a contradiction.

Now let $p>2$ and let $e$ be arbitrary. By the previous proposition there exists a basis $w_{1}, \cdots, w_{e}$ of $G F\left(p^{e}\right)$ over $G F(p)$ such that for all $\beta \in G F\left(p^{e}\right), R_{w}(\beta)$ is symmetric. Let $\sigma_{i}=\left[\alpha_{r s}^{(i)}\right]$ be the matrix $\sigma_{i}=$ $R_{w}\left(w_{i}\right)$. These $e$ matrices of size $e \times e$ over $G F(p)$ have the following properties.

(1) $\alpha_{r s}^{(i)}=\alpha_{s r}^{(i)}$.

(2) $\alpha_{r j}^{(i)}=\alpha_{r i}^{(j)}$ and $\alpha_{i s}^{(j)}=\alpha_{j s}^{(i)}$.

(3) If $\sum f_{i} \sigma_{i}$ is singular for $f_{i} \in G F(p)$, then we must have $f_{1}=$ $f_{2}=\cdots=f_{e}=0$.

Condition (1) follows since $R_{w}\left(w_{i}\right)$ is symmetric and (3) follows since $w_{1}, w_{2}, \cdots, w_{e}$ are a basis of field $G F\left(p^{e}\right)$ over $G F(p)$. Finally $\sigma_{i}\left(w_{j}\right)=$ $R_{w}\left(w_{i}\right) \cdot w_{j}=w_{i} w_{j}$ and hence $\sigma_{i}\left(w_{j}\right)=\sigma_{j}\left(w_{i}\right)$. This yields $\alpha_{r j}^{(i)}=\alpha_{r i}^{(j)}$. The remaining equality in (2) follows from this and symmetry.

Let 


$$
\begin{gathered}
A=g p\left\langle x_{1}, x_{2}, \cdots, x_{e}, y_{1}, y_{2}, \cdots, y_{e}, u, v\right| \\
x_{i}^{p}=y_{i}^{p}=u^{p}=v^{p}=1 \\
u \text { and } v \text { are central } \\
\left(x_{i}, x_{j}\right)=\left(y_{i}, y_{j}\right)=1 \\
\left.\left(y_{i}, x_{j}\right)=u^{\delta_{i j}}\right\rangle
\end{gathered}
$$

where $\delta_{i j}=0$ for $i \neq j$ and $\delta_{i j}=1$ for $i=j$. Clearly $|A|=m^{2} p^{2}$ where $m=p^{e}$ and $A^{\prime}=\langle u\rangle$. Let $\tau_{i}$ act on $A$ by

$$
\begin{aligned}
& u^{\tau_{i}}=u \quad v^{\tau_{i}}=v \\
& y_{j}^{\tau_{i}}=y_{j} v^{\delta_{i j}} \\
& x_{j}^{\tau_{i}}=x_{j}\left(\prod_{r} y_{r}^{\alpha_{j r}^{(i)}}\right) w_{i j}
\end{aligned}
$$

where

$$
w_{i j}=\left(u^{-1} v\right)^{(1 / 2) \alpha_{i i}^{(j)}}
$$

Here division in the exponent is performed modulo $p$.

We show first that $\tau_{i}$ defines an automorphism of $A$. To do this it suffices to show the following.

$$
\begin{aligned}
& \left(x_{j}^{\tau i}\right)^{p}=\left(y_{j}^{\tau i}\right)^{p}=\left(u^{\tau}\right)^{p}=\left(v^{\tau_{i}}\right)^{p}=1 \\
& u^{\tau} i \text { and } v^{\tau_{i}} \text { are central in } A \\
& \left(y_{j}^{\tau i}, y_{k}^{\tau i}\right)=1 \\
& \left(y_{j}^{\tau i}, x_{k}^{\tau i}\right)=\left(u^{\tau}\right)^{\delta_{j k}}=u^{\delta j_{k}} \\
& \left(x_{j}^{\tau}, x_{k}^{\tau i}\right)=1 .
\end{aligned}
$$

Now $A$ has class 2 and $p>2$ so $A$ is regular. Since it is generated by elements of order $p$, it has period $p$. Hence the first equation holds. Since $A$ has class 2 , the next three equations are obvious. We consider the last one now. We have

$$
x_{j}^{\tau i}=x_{j}\left(\Pi_{r} y_{r}^{\alpha_{j r}^{(i)}}\right) w_{i j} \quad x_{k}^{\tau i}=x_{k}\left(\Pi_{r} y_{r}^{\alpha_{k r}^{(i)}}\right) w_{i k}
$$

so

$$
\left(x_{j}^{\tau i}, x_{k}^{\tau i}\right)=\left(x_{j}, y_{j}^{\alpha_{k j}^{(i)}}\right)\left(y_{j k}^{\alpha_{j k}^{(i)}}, x_{k}\right)=u^{\alpha_{j k}^{(i)}-\alpha_{k j}^{(i)}}=1
$$

by (1). Thus $\tau_{i}$ is an automorphism of $A$.

We show now that as an automorphism $\tau_{i}$ has order $p$. Clearly $\tau_{i} \neq 1$. Now $\tau_{i}$ fixes $u$ and $v$ and $y_{j}^{\tau_{i}^{n}}=y_{j} v^{n \delta_{i j}}$. Thus $\tau_{i}^{p}$ fixes $y_{j}$. Finally 


$$
x_{j}^{\tau_{i}^{n}}=x_{j}\left(\Pi y_{r}^{\alpha_{j r}^{(i)}}\right)^{n} v^{\alpha_{j i}^{(i)} n(n-1) / 2} w_{i j}^{n} .
$$

So for $p>2$, $\tau_{i}^{p}$ fixes $x_{j}$ and hence $\tau_{i}$ has order $p$.

We know that $\tau_{i}, \tau_{j} \in$ Aut $A$. We show that $\tau_{i} \tau_{j}=\tau_{j} \tau_{i}$. Clearly $u=u^{\tau_{i} \tau_{j}}=u^{\tau \tau^{\tau} i}, v=v^{\tau_{i} \tau_{j}}=v^{\tau_{j} \tau_{i}}$ and $y_{k}^{\tau_{i} \tau_{j}}=y_{k} v^{\delta_{i k}+\delta_{j k}}=y_{k}^{\tau^{j} \tau_{i}}$. Finally

$$
\begin{aligned}
x_{i}^{\bar{i}^{i \tau} j} & =\left[x_{k}\left(\Pi y_{r}^{\alpha_{k r}^{(i)}}\right) w_{i k}\right]^{\tau_{j}} \\
& =x_{k}\left(\Pi y_{r}^{\alpha_{k r}^{(j)}}\right) w_{j k}\left(\Pi y_{r}^{\alpha_{k r}^{(i)}}\right) w_{i k} v^{\alpha_{k j}^{(i)}}
\end{aligned}
$$

and

$$
\begin{aligned}
x_{k}^{\tau_{j}^{j} j_{i}} & =\left[x_{k}\left(\Pi y_{r}^{\alpha_{k r}^{(j)}}\right) w_{j k}\right]^{\tau_{i}} \\
& =x_{k}\left(\Pi y_{r}^{\alpha_{k r}^{(i)}}\right) w_{i k}\left(\Pi y_{r}^{\alpha_{k r}^{(j)}}\right) w_{j k} v^{\alpha_{k i}^{(j)}} .
\end{aligned}
$$

These two expressions are equal since $\alpha_{k i}^{(j)}=\alpha_{k j}^{(i)}$ by (2).

Let $E=g p\left\langle z_{1}, z_{2}, \cdots, z_{e} \mid z_{i}^{p}=\left(z_{i}, z_{j}\right)=1\right\rangle$ and set $G=A \times{ }_{\tau} E$, the semidirect product of $A$ by $E$, where $\tau: E \rightarrow$ Aut $A$ is the map induced by $z_{i} \rightarrow \tau_{i}$. We note some elementary properties of $G$. Clearly $|G|=m^{3} p^{2}, G^{\prime}=\left\langle y_{1}, y_{2}, \cdots, y_{e}, u, v\right\rangle$ and $\gamma^{2} G=\langle u, v\rangle$ so that $G$ has class 3 . We show that $\mathbb{E}\left(G^{\prime}\right)=G^{\prime}$ so that $\left[G: \mathfrak{C}\left(G^{\prime}\right)\right]=m^{2}$. Since $G^{\prime}$ is abelian we have $\mathfrak{S}\left(G^{\prime}\right) \supseteqq G^{\prime}$. On the other hand if

$$
h=g\left(\prod_{i} x_{i}^{a} i\right)\left(\prod_{i} z_{i}^{b i}\right) \in \mathfrak{S}\left(G^{\prime}\right)
$$

with $g \in G^{\prime}$, then for all $j, 1=\left(y_{j}, h\right)=u^{a_{j}} v^{b_{j}}$ and hence $a_{j}=b_{j}=0$. Thus $\mathfrak{s}\left(G^{\prime}\right)=G^{\prime}$.

Since $[G: A]=m$, we see easily by (3) and Lemma 3.8 (i) that $G / \gamma^{2} G$ has a.c. $m$. Set $B=\left\langle y_{1}, y_{2}, \cdots, y_{e}, z_{1}, z_{2}, \cdots, z_{e}, u, v\right\rangle$. We see that $3(A)=3(B)=\langle u, v\rangle, A^{\prime}=\langle u\rangle$ and $B^{\prime}=\langle v\rangle$. Since $[A: 3(A)]=$ [B: $3(B)]=m^{2}$ we conclude by Lemma 2.3 of [2] that both $A$ and $B$ have a.c. $m$. Let $\chi$ be an irreducible character of $G$ with $\gamma^{2} G \nsubseteq \operatorname{ker} \chi$. Then either $u \notin \operatorname{ker} \chi$ or $v \notin \operatorname{ker} \chi$ or both. If say $u \notin \operatorname{ker} \chi$, then $\chi \mid A$ is faithful on $A^{\prime}$ and hence $\operatorname{deg} \chi \geqq m$. Similarly if $v \notin \operatorname{ker} \chi$. Thus in either case $\operatorname{deg} \chi \geqq m$.

For each integer $t$ set $E_{t}=\left\langle z_{1} x_{1}^{t}, z_{2} x_{2}^{t}, \cdots, z_{e} x_{e}^{t}\right\rangle$ and $J_{t}=\left\langle v u^{t}\right\rangle$ so that $E_{0}=E$. We show that $E_{t}^{\prime} \subseteq J_{t}$. Now

$$
\begin{aligned}
\left(z_{i} x_{i}^{t}, z_{j} x_{j}^{t}\right) & =x_{i}^{-t} z_{i}^{-1}\left(z_{i} x_{i}^{t}\right)^{z_{j} x_{j}^{t}} \\
& =x_{i}^{-t} z_{i}^{-1}\left[z_{i}\left(x_{i}\left(\Pi y_{r}^{\alpha_{i r}^{(j)}}\right) w_{j i}\right)^{t}\right]_{j}^{x_{j}^{t}} \\
& =x_{i}^{-t} \widetilde{z}_{i}^{-1}\left[z_{i} x_{i}^{t}\left(\Pi y_{r}^{\alpha_{i r}^{(j)}}\right)^{t} u^{\alpha_{i i}^{(j)} t(t-1) / 2} w_{j i}^{t}\right]^{x_{j}^{t}} \\
& =x_{i}^{-t} z_{i}^{-1} x_{j}^{-t} z_{i} x_{j}^{t} x_{i}^{t} \cdot\{\}
\end{aligned}
$$

where 


$$
\begin{aligned}
\{\quad\} & =\left[\left(\Pi y_{r}^{\alpha_{i r}^{(j)}}\right)^{t} u^{\alpha_{i i}^{(j)} t(t-1) / 2} w_{j i}^{t}\right]^{x_{j}^{t}} \\
& =\left(\Pi y_{r}^{\alpha_{i r}^{(j)}}\right)^{t} u^{\alpha_{i j}^{(j)} t^{2}} u^{\alpha_{i i}^{(j)} t(t-1) / 2} w_{j i}^{t} .
\end{aligned}
$$

Hence

$$
\begin{aligned}
\left(z_{i} x_{i}^{t}, z_{j} x_{j}^{t}\right) & =x_{i}^{-t}\left(x_{j}^{-t}\right)^{z_{i}} x_{j}^{t} x_{i}^{t} \cdot\{\quad\} \\
& =x_{i}^{-t}\left[x_{j}\left(\Pi y_{r}^{\alpha_{j r}^{(i)}}\right) w_{i j}\right]^{-t} x_{j}^{t} x_{i}^{t} \cdot\{\quad\} \\
& =x_{i}^{-t} w_{i j}^{-t}\left(\Pi y_{r}^{\alpha_{j r}^{(i)}}\right)^{-t} u^{-\alpha_{j j}^{(i)}(t-1) / 2} x_{j}^{-t} x_{j}^{t} x_{i}^{t} \cdot\{\quad\} \\
& =w_{i j}^{-t}\left(\Pi y_{r}^{\alpha_{j r}^{(i)}}\right)^{-t} u^{-\alpha_{j j}^{(i)} t(t-1) / 2} u^{-d_{j i}^{(i)} t^{2}} \cdot\{\},
\end{aligned}
$$

Since $\alpha_{j r}^{(i)}=\alpha_{i r}^{(j)}$ by (2) and since all terms in the last line above commute, it follows that the $y_{r}$ terms drop out. Thus

$$
\left(z_{i} x_{i}^{t}, z_{j} x_{j}^{t}\right)=w_{j i}^{t} w_{i j}^{-t} u^{\left(\alpha_{i i}^{(j)}-\alpha_{j j}^{(i)}\right) t(t-1) / 2} u^{\left(\alpha_{i j}^{(j)}-\alpha_{j i}^{(i)}\right) t^{2}} .
$$

Now $\alpha_{i j}^{(j)}=\alpha_{j j}^{(i)}$ and $\alpha_{j i}^{(i)}=\alpha_{i i}^{(j)}$ by (2) and so

$$
\left(z_{i} x_{i}^{t}, z_{j} x_{j}^{t}\right)=\left[w_{j i}^{2} w_{i j}^{-2} u^{(t+1)}\left(\alpha_{j j}^{\left.(i)-\alpha_{i i}^{(j)}\right)}\right]^{t / 2}\right.
$$

where $t / 2$ is viewed as division in $G F(p)$. Finally using $w_{i j}^{2}=\left(u^{-1} v\right)^{\alpha_{i i}^{(j)}}$ we obtain

$$
\left(z_{i} x_{i}^{t}, z_{j} x_{j}^{t}\right)=\left(v u^{t}\right)^{\left(\alpha_{j j}^{(i)}-\alpha_{i i}^{(j)}\right) t / 2} \in J_{t}
$$

Thus $E_{t}^{\prime} \subseteq J_{t}$.

Set $B_{t}=\left\langle G^{\prime}, E_{t}\right\rangle$. Then $\left[G: B_{t}\right]=m$ and $B_{t}^{\prime}=J_{t}$. The latter follows since $G^{\prime}$ is abelian, $E_{t}^{\prime} \subseteq J_{t}$ and $\left(y_{i}, z_{j} x_{j}^{t}\right)=\left(v u^{t}\right)^{\delta_{i j}}$. We show now that $G$ is a special class 3 group with a.c. $m$. Let $\chi$ be an irreducible nonlinear character of $G$. If $\operatorname{ker} \chi \supseteqq \gamma^{2} G$, then $\chi$ is a character of $G / \gamma^{2} G$ and hence has degree $m$. Assume $\operatorname{ker} \chi \nsupseteq \gamma^{2} G$. As we showed above $\operatorname{deg} \chi \geqq m$. Let $J=\operatorname{ker} \chi \cap \gamma^{2} G$ so that $G$ is a character of $G / J$. If $J=\langle u\rangle$, then $G / J$ has an abelian subgroup $A / J$ of index $m$ and if $J=\left\langle v u^{t}\right\rangle=J_{t}$ then $G / J$ has an abelian subgroup $B_{t} / J$ of index $m$. Thus $\operatorname{deg} \chi \leqq m$ and hence $\operatorname{deg} \chi=m$. This shows that $G$ has a.c. $m$. Since $G$ has class 3 and $\left[G: \mathbb{C}\left(G^{\prime}\right)\right]=m^{2}$, we see that $G$ is a special class 3 group. This completes the proof of the theorem.

5. Special class 2 groups. In this section we study special class 2 groups with a.c. $m\left(m=p^{e}\right)$. As is to be expected, the structure of these groups is less restrictive than in the class 3 case. Let $G$ have a.c. $p^{e}$. We say $G$ is imprimitive if it has a normal subgroup $H$ of index $p$ with a.c. $p^{e-1}$. Otherwise $G$ is primitive. We first note the following. Let $G$ have a.c. $p^{e}$ and let $H \triangle G$ with $[G: H]=p$. If $H$ has a.c. $p^{e-1}$, 
then certainly $e(H)=e(G)-1$. Suppose now that $e(H)=e(G)-1$. If $\phi$ is a nonlinear irreducible character of $H$ and if $\chi$ is a constituent of $\varphi^{*}$, then $\operatorname{deg} \chi=p^{e}$ so $\operatorname{deg} \varphi=p^{e-1}$. Hence $H$ has a.c. $p^{e-1}$. Thus the concepts of imprimitivity as an r.x.e group and as an a.c. $p^{e}$ group are entirely equivalent. We now relate this idea to a certain characteristic subgroup of $G$.

Proposition 5.1. Let $G$ have a.c. $m$ and class 2. Set

$$
S=S(G)=\langle g \in G|(g, G)\left\langle G^{\prime}\right\rangle \text {. }
$$

Then we have the following.

(i) If $[G: S(G)]<m$, then $G$ is a special class 2 group.

(ii) $G$ is primitive if and only if $G=S(G)$.

Proof. (i) Suppose that $G$ has a normal abelian subgroup $A$ of index $m$. If $x \in G-A$, then by Lemma 3.3 (iii), $(x, A)=G^{\prime}$ and hence $(x, G)=G^{\prime}$. Thus $S(G) \cong A$ and $[G: S(G)] \geqq m$, a contradiction.

(ii) We show that $G>S(G)$ if and only if $G$ is imprimitive. Suppose first that $G>S(G)$. Choose subgroup $H$ with $G>H \supseteqq S(G)$ and $[G: H]=p$. Let $\varphi$ be a nonlinear irreducible character of $H$ and let $\chi$ be constituent of $\varphi^{*}$. If $x \in G-H$, then $(x, G)=G^{\prime} \nsubseteq \operatorname{ker} \chi$ and thus $x$ is not in the center of the representation associated with $\chi$. Since $x \in Z_{2}(G)$, this yields $\chi(x)=0$. Thus $\chi$ vanishes off $H$ and so $\chi=\varphi^{*}$. This yields $p \operatorname{deg} \varphi=\operatorname{deg} \varphi^{*}=\operatorname{deg} \chi=p^{e}$ and thus $H$ has a.c. $p^{e-1}$ and $G$ is imprimitive.

Now let $G$ be imprimitive so that $G$ has a normal subgroup $H$ of index $p$ with a.c. $p^{e-1}$. We show that $S(G) \subseteq H$. If not, there exists $x \in G-H$ with $W=(x, G)<G^{\prime}$. Note that $x$ is central modulo $W$ and we have $G / W=(H / W) 3(G / W)$. Since $W<G^{\prime}$ we see that $G / W$ has a.c. $p^{e}$ and that $H / W$ is either abelian or has a.c. $p^{e-1}$. Let $\chi$ be a nonlinear irreducible character of $G / W$. The above implies that $\chi=\varphi^{*}$ for some irreducible character $\varphi$ of $H / W$. Thus

$$
H / W=T(\varphi) \supseteqq(H / W) B(G / W)=G / W,
$$

a contradiction. Therefore $G>H \supseteqq S(G)$.

We now consider an example.

EXAMPLE 5.2. Let $Z$ be an elementary abelian group of order $p^{s+1}$ with $s>0$. Set $k=\left(p^{s+1}-1\right) /(p-1)$ and suppose that $E_{1}, E_{2}, \cdots$, $E_{k}$ are $k$ nonabelian groups of order $p^{3}$. Let $Z_{i}=\left\langle z_{i}\right\rangle$ be the center of $E_{i}$. We define a homomorphism

$$
\tau:=Z_{1} \times Z_{2} \times \cdots \times Z_{k} \longrightarrow Z
$$


by sending each $z_{i}$ onto a generator of the $k$ distinct subgroups of $Z$ of order $p$. Let $N$ be the kernel of $\tau$. Then $N$ is central and hence normal in $E=E_{1} \times E_{2} \times \cdots \times E_{k}$. Set $G=E / N$.

It is clear that $Z=3(G)=G^{\prime}$. Let $\chi$ be a nonlinear irreducible character of $G$ so that $\chi \mid Z=(\operatorname{deg} \chi) \lambda$ with $\lambda \neq 1$. By way of the homomorphism $E \rightarrow G, \chi$ can be viewed as a character of $E$ and as such $\chi=\theta_{1} \theta_{2} \cdots \theta_{k}$ where $\theta_{i}$ is a character of $E_{i}$ and hence has degree 1 or $p$. Moreover $\operatorname{deg} \theta_{i}=p$ if and only if $Z_{i} N / N \nsubseteq \operatorname{ker} \lambda$. Thus there are precisely $\left(p^{s+1}-p^{s}\right) /(p-1)=p^{s}$ such $\theta_{i}$ with $\operatorname{deg} \theta_{i}=p$ and hence $\operatorname{deg} \chi=p^{p^{s}}$. Thus $G$ has a.c. $p^{p^{s}}$.

Now since $|Z|>p$ we have clearly $E_{i} N / N \subseteq S(G)$ for all $i$. Thus $S(G)=G$ and by Proposition 5.1, $G$ is primitive and therefore special. Note finally that if $e=p^{s}$, then $G$ has a.c. $p^{e}$ and $[G: 3(G)]=p^{2 k}$ with $k>e$.

The above example shows that special class 2 groups with arbitrarily large commutator subgroups and central quotients do in fact exist. However the above construction required that we let $m$ get arbitrarily large. We will show in Theorem 5.5 that this is typical of the general situation. We first obtain a generalization of Theorem $B$ of [2].

THEOREM 5.3. Let $G$ be a p-group with $e(G)=e$. Then either $G$ has a normal abelian subgroup of index $p^{e}$ or $G$ has a subgroup $H$ of index $p^{e-1}$ with $[H: 3(H)] \leqq p^{6 e(e+3)}$.

Proof. By Theorom B of [2], there exists subgroups $N$ and $A$ of $G$ with $[G: N]=p^{e}, A=3(N)$ and $[N: A] \leqq p^{3 e(e+2)}$. If $\mathbb{E}(A)>N$, then we can choose subgroup $H$ with $\mathfrak{C}(A) \supseteqq H>N$ and $[H: N]=p$. With this $H$ the result follows. So assume $\mathbb{C}(A)=N$.

Suppose now that $N$ is not normal in $G$. Let $N^{x} \neq N$. If $B=$ $A \cap A^{x}$, then $\mathbb{E}(B) \supseteqq\left\langle N, N^{x}\right\rangle>N$. Since $[N: B] \leqq p^{6(e+2)+e}$, the result follows also in this case.

Thus we can assume that $N \triangle G$ and hence that $A \triangle G$. If $N=$ $A$, then $G$ has a normal abelian subgroup of index $p^{e}$. Hence we can assume that $N$ is nonabelian. Since $N$ is a $p$-group and $A=3(N)$ we can choose subgroup $J$ with $J \subseteq N^{\prime} \cap A$ and $|J|=p$. Set $n=$ $\min \left\{\left[A: \widetilde{夭}_{A}(x)\right] \mid x \notin N\right\}$. We compute as in Lemma 4.4 of [2].

Clearly

$$
r(A) \leqq|N|+(|G|-|N|) / n \text {. }
$$

Now let $\lambda$ be a character of $A$. If $\lambda$ has $p^{e}$ conjugates, then since $T(\lambda) \supseteqq N$ we have $T(\lambda)=N$. Thus if $\chi$ is a constituent of $\lambda^{*}$, then 
there exists character $\eta$ of $N$ with $\chi=\eta^{*}$ and $\lambda$ a constituent of $\eta \mid A$. Since $\operatorname{deg} \chi \leqq p^{e}$, we see that $\operatorname{deg} \eta=1$. Hence $\lambda=\eta \mid A$ and $\operatorname{ker} \lambda \supseteqq N^{\prime} \cap A \supseteqq J$. Thus we see that

$$
s(A) \geqq[A: J] / p^{e}+(|A|-[A: J]) / p^{e-1} .
$$

By Lemma 4.3 of $[2], r(A)=[G: A] s(A)$. Thus

$$
p^{-e}+\left(1-p^{-e}\right) / n \geqq p^{-e-1}+\left(1-p^{-1}\right) p^{1-e} .
$$

Hence

$$
p^{e+1}>p\left(p^{e}-1\right) /(p-1)^{2} \geqq n .
$$

Choose $x \in G-N$ with $\left[A: \widetilde{\complement}_{A}(x)\right]=n$ and set $\left.K=\langle N, x\rangle\right\rangle N$. Then $3(K)=\mathbb{E}_{A}(x)$ so

$$
[N: 3(K)]=[N: A]\left[A: \mathfrak{S}_{A}(x)\right] \leqq p^{3 e(e+2)} p^{e+1}<p^{6 e(e+3)-1} .
$$

If $H$ is chosen with $K \supseteqq H>N$ and $[H: N]=p$, then the result follows.

We now return to our study of class $2 p$-groups with a.c. $m$.

Lemma 5.4. Let $G$ have a.c. $m$ and class 2. Then $G^{\prime}$ and $G / 3(G)$ are both elementary abelian.

Proof. We show that $G^{\prime}$ is elementary abelian by induction on $|G|$. Of course $G^{\prime}$ is abelian since $G^{\prime} \leqq 3(G)$. If $3(G)$ is not cyclic, let $J_{1}$ and $J_{2}$ be two distinct subgroups of $3(G)$ order $p$. By induction $G^{\prime} J_{i} / J_{i}$ has period $p$ and hence so does $G^{\prime}$. Now let $3(G)$ be cyclic. By Lemma 3.4, $G$ has a normal abelian subgroup $A$ with $G / A$ elementary abelian of order $m$. If $x \in G-A$, then $G^{\prime}=(x, A)$ by Lemma 3.3 (iii). Let $y \in A$. Since $x^{p} \in A$ and $G$ has class 2 , we have $(x, y)^{p}=\left(x^{p}, y\right)=1$ and thus $G^{\prime}$ is elementary abelian.

We show now that $G / 3(G)$ is elementary abelian. The quotient is of course abelian. Let $x, y \in G$. Since $G$ has class 2 and $G^{\prime}$ has period $p$, we have $\left(x^{p}, y\right)=(x, y)^{p}=1$. Thus $x^{p} \in 3(G)$ and $G / 3(G)$ has period $p$. This completes the proof.

We will use the following notation throughout this section. Let $W$ be a subgroup of $G^{\prime}$. Set

$$
Z_{W}=\{g \in G \mid(g, G) \subseteq W\}
$$

so that $Z_{W} / W=3(G / W)$. We let $T$ denote a hyperplane in $G^{\prime}$ and $J$ denote a line (that is, $\left[G^{\prime}: T\right]=p$ and $|J|=p$ ). We have $\left[G: Z_{T}\right]=m^{2}$ by Lemma 2.3 of [2] and $S(G)=\left\langle Z_{T}\right|$ all $\left.T\right\rangle$. 
Theorem 5.5. Let $G$ have a.c. $p^{e}$ and class 2. Suppose that $\left|G^{\prime}\right|=$ $p^{s}$ and $[G: 3(G)]=p^{z}$. Then

(i) $z \leqq e(s+1)$ and $s \leqq \frac{1}{2} z(z-1)$

(ii) if $G$ is special, then $z \leqq 18 e^{3}(e+3)^{2}$ and $s<18 e^{2}(e+3)^{2}$.

Proof. (i) Let $T_{1}$ and $T_{2}$ be two hyperplanes in $G^{\prime}$. We show first that $\left[Z_{T_{1}}: Z_{T_{1}} \cap Z_{T_{2}}\right] \leqq p^{e}$. Let $\chi_{i}$ be a nonlinear irreducible character of $G / T_{i}$ for $i=1,2$. Since $\chi_{i}$ vanishes off $Z_{T_{i}}$, we see that $\chi_{1} \chi_{2}$ vanishes off $N=Z_{T_{1}} \cap Z_{T_{2}}$. Also $\operatorname{deg} \chi_{i}=p^{e}$ so that $\chi_{1} \chi_{2} \mid N=p^{2 e} \lambda$ where $\lambda$ is a linear character of $N$. Now let $\theta$ be an irreducible constituent of $\chi_{1} \chi_{2}$ so that $\theta \mid N=(\operatorname{deg} \theta) \lambda$. Then

$$
1 \leqq\left[\chi_{1} \chi_{2}, \theta\right]=(1 /[G: N])\left[\chi_{1} \chi_{2}|N, \theta| N\right]_{N}=p^{2 e}(\operatorname{deg} \theta) /[G: N] .
$$

Since $\operatorname{deg} \theta \leqq p^{e}$ we have $[G: N] \leqq p^{3 e}$ and hence $\left[Z_{T_{1}}: Z_{T_{1}} \cap Z_{T_{2}}\right] \leqq p^{e}$.

Let $T_{1}, T_{2}, \cdots, T_{u}$ be hyperplanes. We show that $\left[G: \bigcap_{1}^{u} Z_{T_{i}}\right] \leqq$ $p^{e(u+1)}$ by induction on $u$. For $u=1,2$ we have result by the above so let $u \geqq 3$. Set $U=\bigcap_{1}^{u-1} Z_{T_{i}}$ so that by induction $[G: U] \leqq p^{e u}$. Hence since $U \subseteq Z_{T_{1}}$ we have

$$
\begin{aligned}
{\left[G: U \cap Z_{T_{u}}\right] } & =[G: U]\left[U: U \cap Z_{T_{u}}\right] \leqq[G: U]\left[Z_{T_{1}}: Z_{T_{1}} \cap Z_{T_{u}}\right] \\
& \leqq p^{e u} p^{e}=p^{e(u+1)}
\end{aligned}
$$

and this follows.

Since $\left|G^{\prime}\right|=p^{s}$, we can find $s$ hyperplanes $T_{1}, T_{2}, \cdots, T_{s}$ with ก $T_{i}=\langle 1\rangle$. Clearly $\bigcap_{1}^{s} Z_{T_{i}}=3(G)$. By the above

$$
p^{z}=[G: 3(G)]=\left[G: \bigcap_{1}^{s} Z_{T_{i}}\right] \leqq p^{e(s+1)}
$$

and hence $z \leqq e(s+1)$. Now let $x_{1}, x_{2}, \cdots, x_{z} \in G$ generate the quotient $G / 3(G)$. We see easily that the commutators $\left(x_{i}, x_{j}\right)$ with $i<j$ generate $G^{\prime}$. Since $G^{\prime}$ is abelian and has period $p$, this yields $s \leqq \frac{1}{2} z(z-1)$ and (i) follows.

(ii) We apply Theorem 5.3. Since $G$ is special we see that $G$ has a subgroup $H$ of index $p^{e-1}$ with $[H: ß(H)] \leqq p^{6 e(e+3)}$. By Lemma 3.3 (iii), $H^{\prime}=G^{\prime}$. If $[H: 3(H)]=p^{t}$ and $\left|H^{\prime}\right|=\left|G^{\prime}\right|=p^{s}$, then as above we have

$$
s \leqq \frac{1}{2} t(t-1)<\frac{1}{2} t^{2} \leqq 18 e^{2}(e+3)^{2} .
$$

Finally by (i) we obtain

$$
z \leqq e(s+1) \leqq 18 e^{3}(e+3)^{2}
$$

and the theorem is proved.

The above result is of course qualitative in nature. The bounds 
are no where near best possible. If $G$ has an abelian subgroup of index $m$, the following example shows that $\left|G^{\prime}\right|$ and $[G: 3(G)]$ can be arbitrarily large for fixed $m$.

EXAMPLE 5.6. Let group $G$ have a.c. $m$ and a normal abelian subgroup $A$ of index $m$. Given integer $k$, set $B=A_{1}+A_{2}+\cdots+A_{k}$, the direct sum of $k$ copies of $A$. Set $H=B \times{ }_{\sigma}(G / A)$ where $G / A$ acts on $B$ in the natural way. If $x \in G / A$ with $x \neq 1$, then $(x, A)=G^{\prime}$. Hence clearly $(x, B)=H^{\prime}$. By Lemma 3.8 (i) we see that $H$ has a.c. $m$. Moreover $\left|H^{\prime}\right|=\left|G^{\prime}\right|^{k}$ and $[B: 3(H)]=[A: 3(G)]^{k}$.

If we now take $G$ to be an extra-special Case $P$ group with $[G: 3(G)]=m^{2}$, then $G$ has a.c. $m$ and $\left|G^{\prime}\right|=p$. Also $G$ is nonspecial so the above construction yields nonspecial groups $H$ with $\left|H^{\prime}\right|$ and $[H: 3(H)]$ arbitrarily large.

Lemma 5.7. Let $G$ and $H$ have class 2 with $\left|G^{\prime}\right|=\left|H^{\prime}\right|$. Suppose that $G$ has a.c. $m$ and $H$ has a.c.n. Let $K$ be the product of $G$ and $H$ with $G^{\prime}$ and $H^{\prime}$ identified. Then $K$ has class 2 and a.c.mn. Also with $G$ and $H$ naturally embedded in $K$ we have $3(K)=3(G) 3(H)$ and $S(K) \supseteqq S(G) S(H)$.

Proof. By Lemma 5.4, $G^{\prime} \cong H^{\prime}$ and so $K$ clearly exists. Let $\chi$ be a nonlinear irreducible character of $K$. By way of the map $E=$ $G \times H \rightarrow K$ we can view $\chi$ as a character of $E$. As such $\chi=\theta \varphi$ where $\theta$ is a character of $G$ and $\varphi$ is one of $H$. In $K, \operatorname{ker} \chi \nsupseteq K^{\prime}$ and thus in $E, \operatorname{ker} \chi \nsupseteq G^{\prime}$ and $\operatorname{ker} \chi \nsupseteq H^{\prime}$. Hence both $\theta$ and $\varphi$ are nonlinear. Thus $\operatorname{deg} \theta=m, \operatorname{deg} \varphi=n$ and $\operatorname{deg} \chi=m n$. Therefore $K$ has a.c. $m n$. The remaining results are obvious.

The following proposition considers minimal special groups.

Proposition 5.8. Let $G$ be a primitive group with a.c. $p^{e}$ and class 2. Suppose that for all $J \subseteq G^{\prime}$ with $|J|=p$ the quotient $G / J$ has an abelian subgroup of index $p^{e}$. Then either $\left|G^{\prime}\right|=p^{2}$ and $p \mid e$ or $\left|G^{\prime}\right|=p^{3}$. Moreover for all $p, e$ (with $p \mid e$ in the first case) such groups exist.

Proof. We show first that $\left|G^{\prime}\right| \leqq p^{3}$. Suppose by way of contradiction that $\left|G^{\prime}\right| \geqq p^{4}$. Let $T_{1}$ and $T_{2}$ be two not necessarily distinct hyperplanes in $G^{\prime}$. Since $\left|G^{\prime}\right| \geqq p^{4}$ we have $\left|T_{1} \cap T_{2}\right| \geqq p^{2}$. Let $J_{1}$ and $J_{2}$ be two distinct subgroups of $T_{1} \cap T_{2}$ of order $p$. By assumption $G / J_{i}$ has an abelian subgroup $A_{i} / J_{i}$ of index $m=p^{e}$. This implies that $S\left(G / J_{i}\right) \subseteq A_{i} / J_{i}$ and so $Z_{T_{1} / J_{i}}=Z_{T_{1}} / J_{i}$ and $Z_{T_{2} / J_{i}}=Z_{T_{2}} / J_{i}$ are both contained in $A_{i} / J_{i}$. This yields $\left(Z_{T_{1}}, Z_{T_{2}}\right) \subseteq J_{1} \cap J_{2}=\langle 1\rangle$. Now $G$ is 
primitive so $G=S(G)=\left\langle Z_{T}\right\rangle$. Since $\left(Z_{T_{1}}, Z_{T_{2}}\right)=\langle 1\rangle$ for all $T_{1}$ and $T_{2}$ we see that $G$ is abelian, a contradiction. Thus $\left|G^{\prime}\right| \leqq p^{3}$.

By Lemma 3.4, we must have $\left|G^{\prime}\right|=p^{2}$ or $p^{3}$. We consider the case $\left|G^{\prime}\right|=p^{2}$ now. Let $T_{0}, T_{1}, \cdots, T_{p}$ be the subgroups of $G^{\prime}$ of order $p$ and set $Z_{i}=Z_{T_{i}}$. Since $G$ is primitive, $G=S(G)=Z_{0} Z_{1} \cdots Z_{p}$. Consider

$$
W=Z_{i} \cap\left(Z_{0} Z_{1} \cdots Z_{i-1} Z_{i+1} \cdots Z_{p}\right) \text {. }
$$

Note that for $i \neq j,\left(Z_{i}, Z_{j}\right) \leqq T_{i} \cap T_{j}=\langle 1\rangle$ so $Z_{i}$ and $Z_{j}$ commute elementwise. Since $W \subseteq Z_{i}$ we see that $\mathbb{E}(W) \supseteq Z_{0} Z_{1} \cdots Z_{i-1} Z_{i+1} \cdots$ $Z_{p}$ and since $W \subseteq Z_{0} Z_{1} \cdots Z_{i-1} Z_{i+1} \cdots Z_{p}$ we see that $\mathbb{C}(W) \supseteqq Z_{i}$. Thus $\mathfrak{E}(W)=G$. Since clearly $W \supseteqq \not(G)$ we have $W=3(G)$. This says that

$$
G / 3(G)=\dot{\amalg}_{0}^{p} Z_{i} / 3(G) \text {. }
$$

Now $\left[G: Z_{i}\right]=p^{2 e}$ so that $\left|Z_{i}\right|=\left|Z_{j}\right|$. If $\left[Z_{i}: 3(G)\right]=p^{f}$ then the above direct product yields

$$
p^{f(p+1)}=[G: 3(G)]=\left[G: Z_{0}\right]\left[Z_{0}: 3(G)\right]=p^{2 e} p^{f}
$$

and hence $2 e=p f$. If $p \neq 2$, then clearly $p \mid e$. If $p=2$, then $f=$ $e \geqq 1$. Clearly $3\left(Z_{0}\right)=3(G)$ and $Z_{0}^{\prime}=T_{0}$. Hence by Lemma 2.3 of [2], $\left[Z_{0}: 3\left(Z_{0}\right)\right]=p^{f}$ is a square. Thus $2 \mid f, f=e$ and the result follows.

We show now that all such groups exist. Consider first $\left|G^{\prime}\right|=p^{2}$ and $e / p=f$, Example 5.2 with $s=1$ yields a group $H$ with a.c. $p^{p}$, $S(H)=H$ and $\left|H^{\prime}\right|=p^{2}$. Let $G$ be the product of $f$ copies of $H$ with their commutator subgroups indentified. By Lemma 5.7 and induction, $G$ has a.c. $p^{f p}=$ a.c. $p^{e}, S(G)=G$ and $\left|G^{\prime}\right|=p^{2}$. If $J \subseteq G^{\prime}$ with $|J|=p$, then $G / J$ has a cyclic commutator subgroup and hence an abelian subgroup of index $p^{e}$. Thus $G$ is the required example.

Now we consider $\left|G^{\prime}\right|=p^{3}$. Let $F$ be the group of Example 3.7 of [2]. Then $\left|F^{\prime}\right|=p^{3},|F|=p^{6}$ and $S(F)=F$. Also it is easy to see that if $J$ is a subgroup of $F^{\prime}$ of order $p$, then $F / J$ has an abelian subgroup of index $p$. Let $G$ be the product of $e$ copies of $F$ with their commutator subgroups identified. Since $F$ has a.c.p, Lemma 5.7 and induction show that $G$ has a.c. $p^{e},\left|G^{\prime}\right|=p^{3}$ and $S(G)=G$. Let $J$ be a subgroup of $G^{\prime}$ of order $p$. Then each factor in $G / J$ has an abelian subgroup of index $p$ so $G / J$ has an abelian subgroup of index $p^{e}$. This completes the proof.

We now apply the above results to improve the bounds in Theorem 5.5 in case $p>e$. 
THEOREM 5.9. Let $G$ be a special class 2 group with a.c.p $p^{e}$ Suppose that $\left|G^{\prime}\right|=p^{s}$ and $[G: 3(G)]=p^{z}$. If $p>e$, then $z \leqq 4 e^{2}$ and $s \leqq 4 e-1$.

Proof. Let $T$ be a hyperplane in $G^{\prime}$. We show first that $p>e$ implies that $Z_{T}$ is abelian. This of course a consequence of Theorem 1.5 and Proposition 1.7. However we can give an alternate inductive proof as follows. Suppose first that $|T| \geqq p^{2}$. Then we can choose distinct subgroups $J_{1}$ and $J_{2}$ of $T$ of order $p$. By induction $Z_{T / J_{i}}=$ $Z_{T} / J_{i}$ is abelian and hence $Z_{T}^{\prime} \subseteq J_{1} \cap J_{2}=\langle 1\rangle$. Thus we need only consider $|T|=1, p$. If $|T|=1$, then certainly $Z_{T}=3(G)$ is abelian. Now let $|T|=p$ so that $\left|G^{\prime}\right|=p^{2}$. Note that groups $G$ with a.c.p $p^{e}$ and $\left|G^{\prime}\right|=p^{2}$ have the property that if $J$ is any subgroup of $G^{\prime}$ of order $p$, then $G / J$ is nonspecial. Hence since $p>e$, Proposition 5.8 and induction easily imply that $G$ is nonspecial. Therefore $Z_{T}$ is contained in an abelian subgroup of $G$ of index $p^{e}$ and thus $Z_{T}$ is abelian.

We show now that $s \leqq 4 e-1$. Suppose first that $G$ is imprimitive. Choose $H \triangle G$ with $[G: H]=p$ and such that $H$ has a.c. $p^{e-1}$. Since $G$ is special, $H$ is special and hence $e>1$. By Lemma 3.3 (iii), $\left|H^{\prime}\right|=\left|G^{\prime}\right|$. By induction $\left|H^{\prime}\right| \leqq p^{4(e-1)-1}$ and so the result follows here. Now let $G$ be primitive so that $G=S(G)=\left\langle Z_{T}\right\rangle$. We assume that $\left|G^{\prime}\right| \geqq p^{4 e}$ and derive a contradiction. Let $T_{1}$ and $T_{2}$ be two not necessarily distinct hyperplanes and let $x \in Z_{T_{1}}$ and $y \in Z_{T_{2}}$. We show that $x$ and $y$ commute. Since each $Z_{T_{i}}$ is abelian of index $p^{2 e}$ we see that $|(x, G)| \leqq p^{2 e}$ and $|(y, G)| \leqq p^{2 e}$. If $(x, G) \cap(y, G)=\langle 1\rangle$, then certainly $(x, y)=1$. Thus we can suppose that $(x, G) \cap(y, G)\rangle\langle 1\rangle$. This yields $|(x, G)(y, G)| \leqq p^{4 e-1}<\left|G^{\prime}\right|$ and thus we can choose hyperplane $T$ with $T \supseteqq(x, G)(y, G)$. Clearly $x, y \in Z_{T}$ and so $x$ and $y$ commute. Since $G=\left\langle Z_{T}\right\rangle$, the above shows that $G$ is abelian, a contradiction. Hence $\left|G^{\prime}\right|<p^{4}$. Finally by Theorem $5.5(\mathrm{i}) z \leqq e(s+1) \leqq 4 e^{2}$ and the result follows.

6. Additional results. We generalize our r.x.1 results in another direction now.

THEOREM 6.1. Let $G$ be a group with the property that every nonlinear irreducible character has prime degree. Suppose further that at least two distinct primes occur. Then there exists primes $p \neq q$ such that $G$ has one of the following two normal series.

(i) $G \underset{p}{>} Q>\underbrace{}_{q^{2}} 3(G)$

with $G / 3(G)$ and $Q$ both nonabelian.

(ii) $G \underset{p}{>} \underbrace{>}_{q} A=3(G) \times R$ 
with both $G / A$ and $Q$ nonabelian. Here $R$ is elementary abelian of order $r^{m}$ for some prime $r$ and $Q / A$ acts irreducibly on it. Also $\left(r^{m}-1\right) /\left(r^{m / p}-1\right)=q$.

Conversely if group $G$ has either of the above structures and if $\chi$ is an irreducible character of $G$, then $\operatorname{deg} \chi=1, p$ or $q$ and all three degrees occur.

We start with two lemmas.

LEMma 6.2. Let $G$ be a group with the property that every nonlinear irreducible character has prime degree. Then every normal subgroup and quotient group of $G$ has this property.

Proof. The result is clear for quotient groups. Let $N \triangle G$ and let $\phi$ be a nonlinear irreducible character of $N$. If $\chi$ is a constituent of $\varphi^{*}$, then $\chi \mid N=a \sum_{1}^{t} \varphi_{i}$ and hence $\operatorname{deg} \chi=a t \operatorname{deg} \varphi$. Since $\operatorname{deg} \chi$ is a prime, $a t=1$ and $\operatorname{deg} \varphi=\operatorname{deg} \chi$ is a prime.

Lemma 6.3. Let G satisfy the hypothesis of Lemma 6.2. Then $G$ is solvable.

Proof. Since this property is inherited by normal subgroups and quotient groups, it suffices to show that $G$ cannot be a nonabelian simple group. Thus suppose $G$ is nonabelian and simple. Let $\chi$ be a nonlinear irreducible character of minimal degree $p$. Since $G$ is simple, $\chi$ is faithful. If $p=2$ and if $x \in G$ is a nonidentity involution, then since $\operatorname{det} \chi=1$ we see that the eigenvalues of $x$ in this representation are both -1 . Hence $3(G) \neq\langle 1\rangle$, a contradiction. Thus $p>2$.

Let $\pi=\{\operatorname{deg} \varphi \mid \varphi$ is irreducible and $\operatorname{deg} \varphi>p\}$. Then $\pi$ is a set of primes and $q \in \pi$ implies that $q>p+1$. If $\pi$ is empty, then $G$ has r.x.1 for prime $p$ and is therefore solvable. Hence we have $|\pi| \geqq 1$. Since $\chi$ is faithful, a result of Blichfeldt ([7] Satz 196) shows that $G$ has an abelian $\mathfrak{S}_{\pi}$ subgroup $H \neq\langle 1\rangle$.

Let $x \in H^{\sharp}$. Then $|C l x|$ is prime to the degree of every irreducible character $\varphi$ of degree different from $p$. By Burnside's Lemma ([7] Satz 168) since $G$ is simple we have $\varphi(x)=0$. If $\rho$ is the regular character of $G$, then we have $0=\rho(x)=\sum \chi_{i}(1) \chi_{i}(x)=1+p \alpha$ where $\alpha$ is an algebraic integer. This is impossible and the result follows.

We now proceed to prove the theorem.

Proof of Theorem 6.1. We know that $G$ is solvable. Choose $A \triangle G$ with $G / A$ extra-special. We show first that $A$ is abelian. If not let $\varphi$ be a nonlinear irreducible character of $A$ and let $\chi$ be a 
constituent of $\varphi^{*}$. Then $\chi \mid A=a \sum_{1}^{t} \varphi_{i}$ and $\operatorname{deg} \chi=a t \operatorname{deg} \varphi$. Since $\operatorname{deg} \chi$ is a prime we must have $a=t=1$. Let $\theta$ be a nonlinear irreducible character of $G / A$ viewed as one of $G$. Then $\chi_{\theta}$ is irreducible (see Lemma 5.5 of [2]) and $\operatorname{deg} \chi \theta=(\operatorname{deg} \chi)(\operatorname{deg} \theta)$ is not a prime, a contradiction.

If $G / A$ is a Case $P$ quotient for prime $p$, then by Ito's Theorem the degrees of the irreducible characters of $G$ are powers of $p$ which is not the case. Thus $G / A$ is Case $Q$. Let $Q / A$ be the normal Sylow $q$-subgroup of $G / A$. Since $G / A$ has an irreducible character of degree $[G: Q]$ we see that $G / Q$ is cyclic of prime order $p \neq q$.

Note that $Q$ is nonabelian. Otherwise $G$ would have r.x.1 for prime $p$. Now $G / A$ has trivial center so $3(G) \subseteq A \subseteq Q$. We show that $3(Q)=3(G)$. Clearly $3(G) \subseteq 3(Q)$. If $x \in 3(Q)-3(G)$, then there exists $y \in G$ with $(x, y) \neq 1$. Now $Q$ is nonabelian and $z=(x, y) \neq 1$ so there exists a nonlinear irreducible character $\varphi$ of $Q$ with $z \notin \operatorname{ker} \varphi$. As above, there exists an irreducible character $\chi$ of $G$ with $\chi \mid G=\varphi$. Since $x$ is in the center of the representation associated with $\varphi$ and since $\chi \mid Q=\varphi$ we see that $(x, y) \in \operatorname{ker} \chi \cap Q=\operatorname{ker} \varphi$, a contradiction. Hence $3(G)=3(Q)$.

Case 1. $|Q / A| \geqq q^{2}$. Let $\lambda \in \hat{A}$. Then clearly $[G: T(\lambda)]$ is 1 or a prime. If $|Q| A \mid \geqq q^{2}$, then the only subgroup of $G / A$ having prime index is $Q / A$. Hence $T(\lambda) \supseteqq Q$. This implies that $\Im_{G}(A) \supseteqq Q$ and hence $Q$ is nilpotent of class 2 . Let $\varphi$ be a nonlinear irreducible character of $Q$ and let $W=W(\varphi)$ denote the subgroup of $Q$ mapping into the center of the representation. Since clearly deg $\varphi=q$ we have $[Q: W]=$ $q^{2}$ by Lemma 2.3 of [2] and also $W \supseteqq A$. Now $T(\varphi)=G$ so $W / A \triangle G / A$. Hence $W(\varphi)=A$ and $[Q: A]=q^{2}$. We saw above that $A \subseteq B(Q)$. This clearly implies that $A=3(Q)=3(G)$ and $G$ satisfies (i).

We assume now that $|Q / A|=q$. Since $Q$ is nonabelian, $\mathfrak{\mho}_{G}(A) \nsupseteq Q$ and hence $\mathfrak{夭}_{G}(A)=A$. Suppose $A=M \times N$ with $M \triangle G$ and $N \triangle G$ and $N, M \neq\langle 1\rangle$. We show that either $M$ or $N$ is central in $G$. Say $N \nsubseteq 3(G)=3(Q)$. Choose $\lambda \in \hat{N}$ so that $T(\lambda) \cap Q=A$. If $\rho \in \hat{M}$, then $T(\lambda \mu)=T(\lambda) \cap T(\mu)$ and $[G: T(\lambda \mu)]$ is a prime. Hence $T(\mu) \supseteq T(\lambda)$ and so $\mathfrak{\mho}_{G}(M) \supseteqq T(\lambda)$. Since $\mathfrak{\mho}_{G}(M) \triangle G$, this implies that $\mathfrak{\mho}_{G}(M)=G$ and $M \cong 3(G)$. In particular we see that precisely one Sylow subgroup of $A$ is noncentral. Hence $A / 3(G)$ in an $r$-group for some prime $r$.

Case 2. $q \neq r$. Since $Q / A$ is cyclic of prime order, we can write $A=3(Q) \times R$ where $Q / A$ acts fixed point free on $R$ by Lemma 1.2. Also $3(Q)=3(G)$ and $R \triangle G$ since $Q \triangle G$. Let $\lambda$ be a nonprincipal 
linear character of $R$. Then $T(\lambda) \cap Q=A$ and $[G: T(\lambda)]$ is a prime. Hence $|T(\lambda) / A|=p$. Thus $G / A$ acts half transitively but non fixed point free on $\hat{R}$. By Theorem I of [3], $\hat{R}$ is elementary abelian and $G / A$ acts irreducibly on it. Let $\bar{G}=G / A, \bar{Q}=Q / A$ and let $\bar{P}=\Im_{p}(\bar{G})$. Let $W$ be an nonidentity irreducible $\bar{Q}$-submodule of $\hat{R}$. If $\lambda \in W^{\sharp}$, then $\bar{G}=\bar{Q}(T(\lambda) / A)$ and thus $W$ is a $\bar{G}$-module. Hence $\bar{Q}$ acts irreducibly on $\hat{R}$.

We view $\hat{R}$ as a vector space over $G F(r)$ of dimension $m$ and we find $\operatorname{dim} \widetilde{夭}_{\hat{R}}(\bar{P})$. This dimension is clearly invariant under field extension so we can extend to the algebraic closure $F$ of $G F(r)$. If $\bar{Q}=\langle x\rangle$, then since $\hat{R}$ is an irreducible $\bar{Q}$-module, all eigenvalues of $x$ are distinct and not equal to 1 . Let $S$ be an irreducible $\bar{G}$-submodule of $F \otimes \hat{R}$. By Clifford's theorem, this representation restricted to $\bar{Q}$ breaks up into either $p$ distinct conjugates or all equivalent representations. If the latter occured then since all eigenvalues of $x$ are distinct, $\operatorname{dim} S=1$ and hence $\bar{Q}=\bar{G}^{\prime}$ is in the kernel. This contradicts the fact that $x$ has no eigenvalue equal to 1 . Thus the former case must always occur. From this we see easily that $p \mid m$ and $\operatorname{dim} \sqrt{5}_{\hat{R}}(\bar{P})=m / p$.

Now $\bar{G}$ contains $q$ conjugate subgroups $\bar{P}_{1}, \cdots, \bar{P}_{q}$ of order $p$. We have $\bar{\Im}_{\hat{R}}\left(P_{i}\right) \cap \overline{\mathbb{E}}_{\hat{R}}\left(P_{j}\right)=\langle 1\rangle$ for $i \neq j$ and $\hat{R}=\bigcup \mathfrak{\mho}_{\hat{R}}(\bar{P})$. Since $\left|\mathfrak{C}_{\hat{R}}\left(\bar{P}_{i}\right)\right|=r^{m / p}$ we obtain from this disjoint union $\left(r^{m}-1\right)=q\left(r^{m / p}-1\right)$. Finally since $\hat{R}$ is elementary abelian and $\bar{Q}$ acts irreducibly, we see that the same is true for $R$. Thus $G$ satisfies (ii).

Case 3. $q=r$. Here $Q$ is clearly nilpotent. Let $R=\mathfrak{S}_{r}(A)$. As above we have

$$
\hat{R}=\mathfrak{夭}_{\hat{R}}(\bar{Q}) \cup \bigcup_{1}^{q} \mathfrak{夭}_{\hat{R}}\left(\bar{P}_{i}\right) .
$$

Let $W=\mathfrak{\Im}_{\hat{R}}(\bar{G})$ and set

$$
[\hat{R}: W]=r^{m},\left[\Im_{\hat{R}}(\bar{Q}): W\right]=r^{a} \text { and }\left[\complement_{\hat{R}}\left(\bar{P}_{i}\right): W\right]=r^{b} \text {. }
$$

Note since all the $\bar{P}_{i}$ are conjugate this is well defined. Now

$$
(\hat{R}-W)=\left(\mathfrak{\Im}_{\hat{R}}(\bar{Q})-W\right) \cup \bigcup_{1}^{q}\left(\mathfrak{\Im}_{\hat{R}}\left(\bar{P}_{i}\right)-W\right)
$$

is a disjoint union so

$$
r^{m}-1=r^{a}-1+q\left(r^{b}-1\right)
$$

and since $r=q, r^{m}-r^{a}=r^{b+1}-r$. Again since the union is disjoint, we have $a+b \leqq m$ and $2 b \leqq m$. Finally $m>a$ since $\mathfrak{F}_{G}(A)=A$ and hence the above equation yields $m=b+1, a=1$. Since $2 b \leqq m$ we have $m=2$ and $b=1$.

Since $m=2, a=1$ we have $\left[\hat{R}: \Im_{\hat{R}}(\bar{Q})\right]=q$. Thus $|(Q, R)|=q$ 
and $Q^{\prime}$ is cyclic of order $q$. This shows that $[Q: 3(Q)]=q^{2}$ by Lemma 2.3 of [2]. Thus $G$ satisfies (i). (Note, the difference between Cases 1 and 3 is that in the former $G / Q$ acts irreducibly on $Q / 3(G)$ and in the latter it does not.)

We show now that groups with structure (i) or (ii) have characters of degree 1, $p$ and $q$ only. Let $G$ satisfy (i) and let $\chi$ be an irreducible character of $G$. By Ito's Theorem $\operatorname{deg} \chi \mid p q^{2}$ and also $(\operatorname{deg} \chi)^{2} \leqq$ $[G: 3(G)]=p q^{2}$. Since $G / 3(G)$ is nonabelian we see easily that $p \leqq q+1$. This yields $\operatorname{deg} \chi=1, p$ or $q$. Since $G / 3(G)$ is nonabelian, it has a character of degree $p$ and since $Q$ is nonabelian it has a character of degree $q$. Thus $G$ does not have a.c. $p$ or a.c. $q$ and hence $G$ has characters of degree $1, p$ and $q$.

Now let $G$ satisfy (ii) and let $\chi$ be an irreducible character of $G$. By Ito's theorem, $\operatorname{deg} \chi \mid p q$ and hence $\operatorname{deg} \chi=1, p, q$ or $p q$. We show that the latter cannot occur. If $\operatorname{deg} \chi=p q$ and $\chi \mid A=a \sum_{1}^{t} \lambda_{i}$ then $a t=p q$ and also $a^{2} t \leqq p q$. Thus $a=1$ and $t=p q$. Let $\lambda=\lambda_{1}$ and write $\lambda=\eta \varepsilon$ where $\eta \in \widehat{3(G)}$ and $\varepsilon \in \hat{R}$. This implies that $A=T(\lambda)=$ $T(\varepsilon)$. As in our Case 2 computation above, we see that $\bigcup_{1}^{q} \mathbb{E}_{\hat{R}}\left(\bar{P}_{i}\right)$ is a disjoint union and $\left|\mathfrak{\Im}_{\hat{R}}\left(\bar{P}_{i}\right)\right|=r^{m / p}$. Hence $\left|\bigcup_{1}^{q} \mathfrak{\Im}_{R}\left(P_{i}\right)\right|=q\left(r^{m / p}\right)+1=$ $r^{m}$. Thus for every $\varepsilon \in \hat{R}$ we have $T(\varepsilon)>A$, a contradiction and $\operatorname{deg} \chi \neq p q$. Now $G / A$ being nonabelian has a character of degree $p$ and $Q$ has a character of degree $q$. Thus $G$ has characters of degree $1, p$ and $q$. This completes the proof of the theorem.

The following are essentially canonical examples of the above.

EXAmple 6.4. First let $Q$ be a nonabelian group of order $q^{3}$. If $q=2$, let $Q$ be the quaternion group and if $q>2$, let $Q$ have period $q$. As is well known, the group of automorphisms of $Q$, fixing $3(Q)$, is isomorphic to $S P(2, q)=S L(2, q)$ and hence has order $q(q-1)(q+1)$. If we choose prime $p$ with $p \mid(q-1)(q+1)$ then we can find an appropriate automorphism group $P$ of $Q$ of order $p$. Clearly $G=Q \times{ }_{\sigma} P$ satisfies (i).

Now suppose we are given primes $p, q, r$ with $p \neq q$ and $\left(r^{m}-1\right) /\left(r^{m / p}-1\right)=q$. Let $R$ be the additive group of $G F\left(r^{m}\right)$. Since $q \mid\left(r^{m}-1\right)$ we see that the multiplicative group of $G F\left(r^{m}\right)$ has an element $\zeta$ of order $q$. Since $p \mid m$ we see that $G F\left(r^{m}\right)$ has a field automorphism $\sigma$ of order $p$. Let $\bar{G}$ be the set of automorphisms of $R$ given by $x \rightarrow \zeta^{i} \cdot \sigma^{j}(x)$. We see easily that $\bar{G}$ is a group of order $p q$ with a normal subgroup of order $q$. It is nonabelian since the fixed field of $\sigma$ has size $r^{m / p}$ and clearly $q>r^{m / p}$. Thus $G=R \times{ }_{\rho} \bar{G}$ satisfies (ii). 
An interesting corollary to Theorem 6.1 is the following.

CoROLlaRY 6.5. Let $G$ have r.b.3, that is every irreducible character of $G$ has degree at most 3. Then either $G$ has a normal abelian subgroup of index $\leqq 3$ or $G / 3(G)$ is isomorphic to one of the following groups.

(i) the elementary abelian group of order 8

(ii) the two groups of order 27 and period 3

(iii) the symmetric and alternating groups on 4 letters

(iv) the dihedral group of order 18 having an elementary abelian Sylow 3-subgroup.

Proof. If $G$ is abelian, the result is clear. If $G$ has a.c. 2 or a.c.3, then by Theorem $\mathrm{C}$ of [2] either $G$ has a normal abelian subgroup of index $\leqq 3$ or $G / 3(G)$ has order 8 or 27 . Since we can assume that $G / 3(G)$ has no cyclic subgroup of index $\leqq 3$, we obtain (i) and (ii).

We assume now that $G$ has characters of degree 2 and 3 and thus Theorem 6.1 applies. If $p=3, q=2$, then case (ii) of that theorem cannot occur since $G / A$ is nonabelian. Since $Q$ is nonabelian in case (i) we see that $Q / 3(G)$ is type $(2,2)$ and hence $G / 3(G)$ is isomorphic to the alternating group $A_{4}$.

Now let $p=2, q=3$. If $G$ is case (i), then as above $Q / 3(G)$ is type $(3,3)$. Let $x, y \in Q$ generate $Q / 3(G)$. Then $(x, y) \in Z(G)$ and $(x, y) \neq 1$. Since the action of $G / Q$ on $G / B(G)$ is nontrivial and preserves this commutator, we see easily that the action must be dihedral and we obtain (iv). If $G$ is case (ii), then $\left(r^{m}-1\right) /\left(r^{m / 2}-1\right)=3$ and so $r^{m / 2}=2$. Thus $G / 3(G)$ is the extension of a $(2,2)$ group by the nonabelian group of order 6 acting faithfully. Since this group has no normal 3-complement, Burnside's transfer theorem implies that the normalizer of a Sylow 3-subgroup contains an element of order 2 . Hence the extension is split and $G / 3(G) \cong S_{4}$, the symmetric group on 4 letters.

We close with a result which generalizes Theorem 3.5(i).

THEOREM 6.6. Let $p^{e}$ be a fixed power of $p$ with $e>1$ and let $G$ be a group with a nonabelian Sylow p-subgroup. Suppose further that if $\chi$ is a nonlinear irreducible character of $G$, then $p^{e} \mid \operatorname{deg} \chi$ and $p^{e+1} \nmid \operatorname{deg} \chi$. Then $G$ is the direct product of $\mathfrak{S}_{p}(G)$ with an. abelian $p^{\prime}$-group.

Proof. By induction on $|G|$. By Theorem 2.5 (i), $G$ has a normal $p$-complement $K$. Let $P$ be a Sylow $p$-subgroup of $G$. If $P \triangle G$, then $G=P \times K$ and clearly $K$ must be abelian. Suppose $G$ has a 
proper normal subgroup $H$ with $p \nmid[G: H]$. Let $\varphi$ be a nonlinear irreducible character of $H$ and let $\chi$ be a constituent of $\varphi^{*}$. Then $\chi \mid H=a \sum_{1}^{t} \varphi_{i}$ and $\operatorname{deg} \chi=a t \operatorname{deg} \varphi$. Since $a t \mid[G: H]$ we have $|\operatorname{deg} \varphi|_{p}=|\operatorname{deg} \chi|_{p}=p^{e}$. By induction, $P \triangle H$ and since $P$ is characteristic in $H, P \triangle G$ and the result follows.

We assume now that $K \neq\langle 1\rangle$ and that $G$ has no proper normal subgroups of $p^{\prime}$ index and we obtain a contradiction. Let $\lambda$ be a nonprincipal linear character of $K$ which has a linear extension $\mu$ on $G$. Then $G / \operatorname{ker} \mu$ is abelian and not a $p$-group and thus some $H$ as above exists. Since this cannot happen, we see that if $\varphi \neq 1$ is any irreducible character of $K$, then $\varphi^{*}$ has no linear constituents. We show now that $T(\varphi) \triangle G$ and that $G / T(\varphi)$ is elementary abelian of order $p^{e}$.

Note that $G / K \cong P$ is nonabelian and has a.c.p. Let $\chi$ be a constituent of $\varphi^{*}$. Then $\chi \mid K=a \sum_{1}^{t} \varphi_{i}$ and so $t \mid p^{e}$. This yields $[G / K: T(\varphi) / K] \leqq p^{e}$ and $T(\varphi) \triangle G$ by Lemma 3.3 (iii). Now let $\xi$ be an irreducible character of $T(\phi)$ with $\xi \mid K=b(\xi) \cdot \varphi$. Clearly $T(\xi)=$ $T(\varphi)$ and hence $\xi^{*}$ is irreducible. Since $\xi^{*}$ is a constituent of $\varphi^{*}$, it is nonlinear and thus $t b(\xi)=p^{e}$. In particular, for all such choices of $\xi, b(\xi)$ is the same. Now by Theorem 6 of [1], there exists $\xi_{0}$ with $b\left(\xi_{0}\right)=1$. Thus $t=p^{e}$ and for all such $\xi, b(\xi)=1$. Let $\beta$ be an irreducible character of $T(\varphi) / K$ viewed as one of $T(\varphi)$. Then $\xi=\xi_{0} \beta$ is irreducible and $\xi \mid K=\beta(1) \cdot \varphi$. Therefore $\beta(1)=1$ and $T(\varphi) / K$ is abelian. As in the latter part of the proof of Lemma 3.4, we see that $G / T(\varphi)$ is elementary abelian of order $p^{e}$.

Now let $x \in K$ with $x \neq 1$ and suppose that $\left[P: \mathfrak{c}_{P}(x)\right] \leqq p^{e}$. We show that $\mathfrak{\Im}_{P}(x) \triangle P$ and $P / \mathfrak{C}_{P}(x)$ is elementary abelian of order $p^{e}$. Let $\tau$ be a nonprincipal linear character of $\langle x\rangle$. Clearly $\mathfrak{S}_{P}(x)$ fixes $\tau$ and hence $\widetilde{ }_{P}(x)$ fixes $\widetilde{\tau}$ (induction to $K$ ). Since the degree of $\widetilde{\tau}$ is prime to $p$ we see that $\mathfrak{c}_{P}(x)$ fixes some irreducible constituent $\varphi$ of $\tilde{\tau}$. Clearly $\varphi \neq 1$ so $T(\varphi) \supseteqq K \Im_{P}(x)$ and $[G: T(\varphi)]=p^{e}$. Hence $T(\varphi)=$ $K \mathfrak{\subseteq}_{P}(x)$ and $G / T(\varphi) \cong P / \mathfrak{S}_{P}(x)$ is elementary abelian of order $p^{e}$.

Let $K$ have $k$ nonprincipal irreducible characters and hence $k$ nonidentity classes. We have shown that in the action of $P$ on the characters of $K$ we have $1+k / p^{e}$ orbits. Hence by Brauer's Lemma, the same is true for the action of $P$ on the classes of $K$. In particular there must exist a class, say $C l y$, belonging to an orbit of size $\leqq p^{e}$ with $y \neq 1$. Let $S$ be the subgroup of $P$ fixing this class so that $[P: S] \leqq p^{e}$. Since $|C l y|$ is prime to $p$, there exists $x \in C l y$ with $S \subseteq \mathfrak{\complement}_{P}(x)$. Thus $\left[P: \mathfrak{\complement}_{P}(x)\right] \leqq p^{e}$ and by the above $P / \mathfrak{C}_{P}(x)$ is elementary abelian of order $p^{e}$. Clearly $S=\widetilde{C}_{P}(x)$. Since $S \triangle P$ we see that $P / S$ acts on $\mathfrak{F}_{K}(S) \neq\langle 1\rangle$. As above, if $z \in \mathfrak{F}_{K}(S)$ with $z \neq 1$, then $\mathfrak{S}_{P}(z)=S$. Hence $P / S$ acts fixed point free on $\mathfrak{F}_{K}(S)$, a contradiction since $P / S$ is elementary abelian of order $p^{e} \geqq p^{2}$. This completes the proof. 


\section{REFERENCES}

1. P. X. Gallagher, Group characters and normal Hall subgroups, Nagoya Math. J. 21 (1962), 223-230.

2. I. M. Isaacs and D. S. Passman, A characterization of groups in terms of the degrees of their characters, Pacific J. Math. 15 (1965), 877-903.

3. — Half-transitive automorphism groups, Canad. J. Math. 18 (1966), 12431250 .

4. D. S. Passman, Groups whose irreducible representations have degrees dividing $p^{2}$, Pacific J. Math. 17 (1966), 475-496.

5. - Groups with normal, solvable Hall p'-subgroups, Trans. Amer. Math. Soc. 123 (1966), 99-111.

6. Character kernels of discrete groups, Proc. Amer. Math. Soc. 17 (1966), 487-492.

7. A. Speiser, Die Theorie der Gruppen von Endlicher Ordnung, $4^{\text {th }}$ ed., Birkhauser, Basel, 1956.

8. O. Taussky and $\mathrm{H}$. Zassenhaus, On the similarity transformation between a matrix and its transpose, Pacific J. Math. 9 (1959), 893-896.

Received December 28, 1966.

UNIVERSITY OF CHICAGO

YALE UNIVERSITY 


\section{PACIFIC JOURNAL OF MATHEMATICS}

\section{EDITORS}

\author{
H. ROYDEN \\ Stanford University \\ Stanford, California
}

\author{
J. P. JANS \\ University of Washington \\ Seattle, Washington 98105
}

J. DugundJI

Department of Mathematics

Rice University

Houston, Texas 77001

RICHARD ARENS

University of California

Los Angeles, California 90024

\section{ASSOCIATE EDITORS}
E. F. BECKENBACH
B. H. NeumanN
F. WOLF
K. YOSIDA

\section{SUPPORTING INSTITUTIONS}

\author{
UNIVERSITY OF BRITISH COLUMBIA \\ CALIFORNIA INSTITUTE OF TECHNOLOGY \\ UNIVERSITY OF CALIFORNIA \\ MONTANA STATE UNIVERSITY \\ UNIVERSITY OF NEVADA \\ NEW MEXICO STATE UNIVERSITY \\ OREGON STATE UNIVERSITY \\ UNIVERSITY OF OREGON \\ OSAKA UNIVERSITY \\ UNIVERSITY OF SOUTHERN CALIFORNIA
}

\author{
STANFORD UNIVERSITY \\ UNIVERSITY OF TOKYO \\ UNIVERSITY OF UTAH \\ WASHINGTON STATE UNIVERSITY \\ UNIVERSITY OF WASHINGTON \\ AMERICAN MATHEMATICAL SOCIETY \\ CHEVRON RESEARCH CORPORATION \\ TRW SYSTEMS \\ NAVAL ORDNANCE TEST STATION
}

\footnotetext{
Mathematical papers intended for publication in the Pacific Journal of Mathematics should be in typed form or offset-reproduced, double spaced with large margins. Underline Greek letters in red, German in green, and script in blue. The first paragraph or two must be capable of being used separately as a synopsis of the entire paper. It should not contain references to the bibliography. Manuscripts may be sent to any one of the four editors. All other communications to the editors should be addressed to the managing editor, Richard Arens, University of California, Los Angeles, California 90024.

Each author of each article receives 50 reprints free of charge; additional copies may be obtained at cost in multiples of 50 .

The Pacific Journal of Mathematics is published monthly. Effective with Volume 16 the price per volume (3 numbers) is $\$ 8.00$; single issues, $\$ 3.00$. Special price for current issues to individual faculty members of supporting institutions and to individual members of the American Mathematical Society: $\$ 4.00$ per volume; single issues $\$ 1.50$. Back numbers are available.

Subscriptions, orders for back numbers, and changes of address should be sent to Pacific Journal of Mathematics, 103 Highland Boulevard, Berkeley 8, California.

Printed at Kokusai Bunken Insatsusha (International Academic Printing Co., Ltd.), 7-17, Fujimi 2-chome, Chiyoda-ku, Tokyo, Japan.

PUBLISHED BY PACIFIC JOURNAL OF MATHEMATICS, A NON-PROFIT CORPORATION

The Supporting Institutions listed above contribute to the cost of publication of this Journal, but they are not owners of publishers and have no responsibility for its content or policies.
} 


\section{Pacific Journal of Mathematics}

\section{Vol. 24, No. $3 \quad$ July, 1968}

Duane W. Bailey, On symmetry in certain group algebras ............ 413

Lawrence Peter Belluce and Surender Kumar Jain, Prime rings with a one-sided ideal satisfying a polynomial identity ................ 421

L. Carlitz, A note on certain biorthogonal polynomials ............. 425

Charles O. Christenson and Richard Paul Osborne, Pointlike subsets of a manifold ......................................... 431

Russell James Egbert, Products and quotients of probabilistic metric

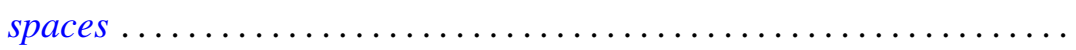

Moses Glasner, Richard Emanuel Katz and Mitsuru Nakai, Bisection into small annuli ..................................... 457

Karl Edwin Gustafson, A note on left multiplication of semigroup

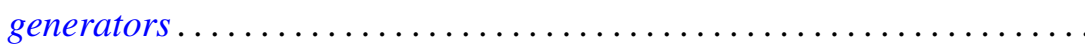

I. Martin (Irving) Isaacs and Donald Steven Passman, A characterization of groups in terms of the degrees of their characters. II ............. 467

Howard Wilson Lambert and Richard Benjamin Sher, Point-like

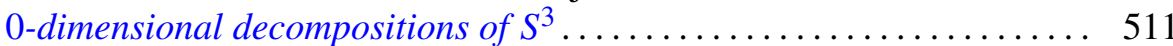

Oscar Tivis Nelson, Subdirect decompositions of lattices of width two ..... 519

Ralph Tyrrell Rockafellar, Integrals which are convex functionals . . . . . . . 525

James McLean Sloss, Reflection laws of systems of second order elliptic differential equations in two independent variables with constant coefficients ...

Bui An Ton, Nonlinear elliptic convolution equations of Wiener-Hopf type in a bounded region

Daniel Eliot Wulbert, Some complemented function spaces in $C(X)$

Zvi Ziegler, On the characterization of measures of the cone dual to a generalized convexity cone. 